\title{
ELECTROPHYSIOLOGICAL STUDIES OF IDENTIFYING AND MODULATING THE PREPARATORY STRATEGIC PROCESSES INVOLVED IN EPISODIC MEMORY RETRIEVAL
}

Dissertation presented to the Faculty of the Graduate School

University of Missouri

In Partial Fulfillment of the Requirements for the Degree

Doctor of Philosophy

\author{
By \\ MASON H. PRICE, M.A. \\ Dr. Jeffrey D. Johnson, Dissertation Supervisor \\ December, 2018
}


The undersigned, appointed by the dean of the Graduate School, have examined the dissertation entitled

\section{ELECTROPHYSIOLOGICAL STUDIES OF IDENTIFYING AND MODULATING THE PREPARATORY STRATEGIC PROCESSES INVOLVED IN EPISODIC MEMORY RETRIEVAL}

presented by Mason H. Price,

a candidate for the degree of doctor of philosophy, and hereby certify that, in their opinion, it is worthy of acceptance.

Associate Professor Jeffrey Johnson (Chair)

Associate Professor Steven Hackley

Associate Professor Shawn Christ

Assistant Professor Roxana Botezatu 


\section{ACKOWLEDGMENTS}

I would like to thank Dr. Jeff Johnson for his support during my graduate studies. I appreciate the time and resources he has invested in my scholarly training and the knowledge he has shared with me, which will be invaluable as I continue my career as an academic researcher. I would also like to thank my committee members, Drs. Botezatu, Christ, and Hackley for their feedback and comments during my thesis and dissertation. I'd like to thank Dr. Christ again for supporting me early on in my graduate education, exposing me first-hand to the clinical brain imaging world. Lastly, l'd like to extend thanks to the faculty and staff of the Department of Psychological Sciences and in particular, the Cognition and Neuroscience area. 


\section{TABLE OF CONTENTS}

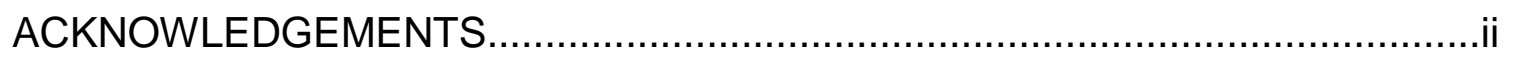

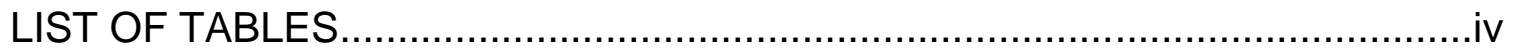

LIST OF FIGURES.............................................................................

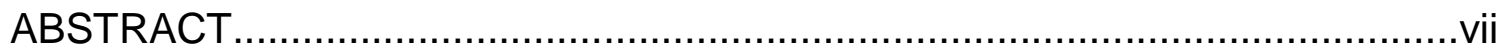

CHAPTER

1. GENERAL

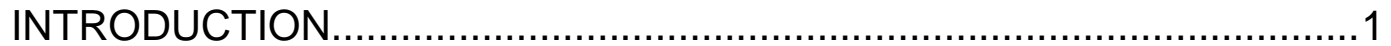

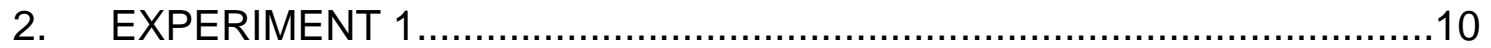

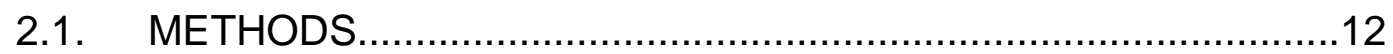

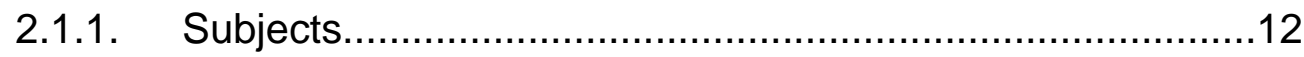

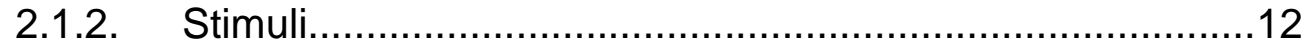

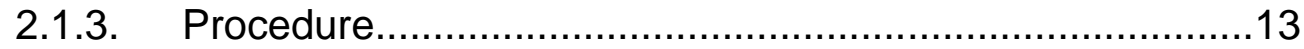

2.1.4. EEG Acquisition and Processing...................................15

2.1.5. EEG Analysis.............................................................16

2.2. RESULTS ...................................................................... 17

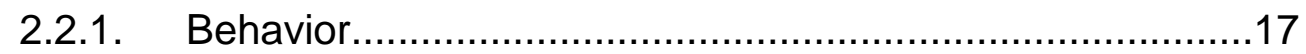

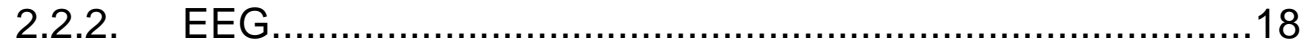

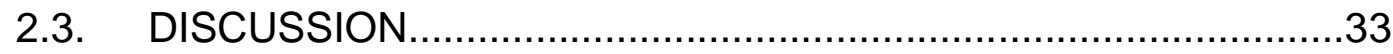

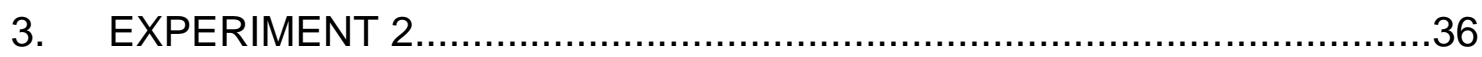

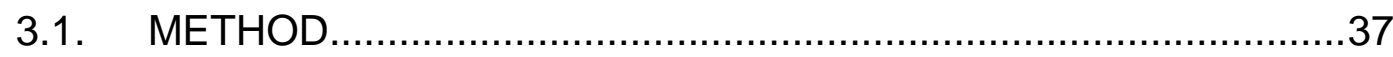

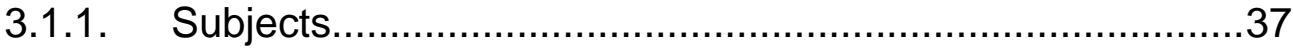

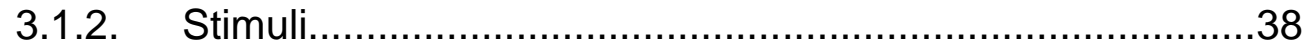

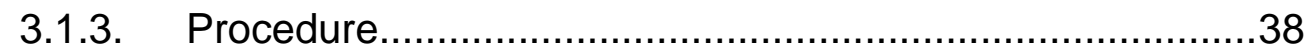

3.1.4. EEG Acquisition and Processing..................................... 41

3.1.5. EEG Analysis....................................................... 41

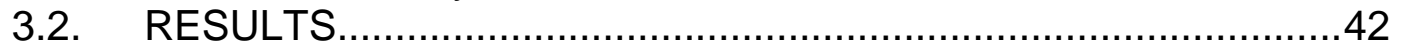

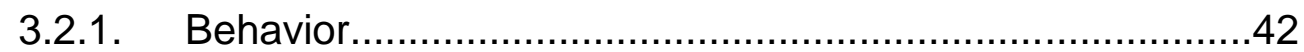

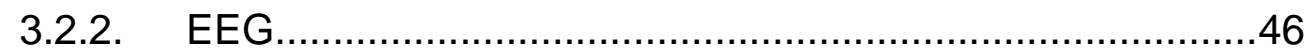

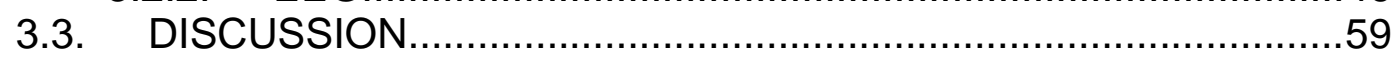

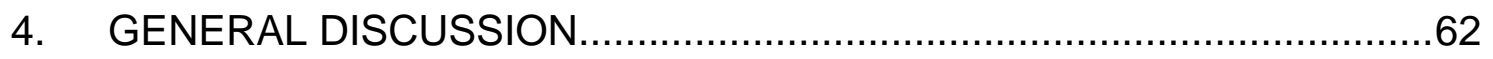

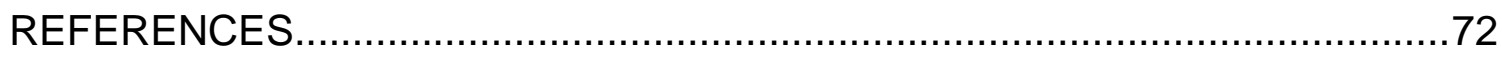

VITA 


\section{LIST OF TABLES}

Table

Page

1. Behavioral Measures for Experiment 1 ..............................................17

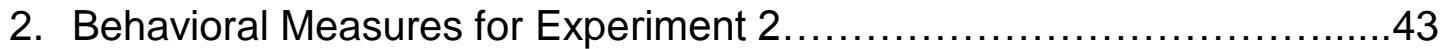




\section{LIST OF FIGURES}

Figure

Page

1. Pre-stimulus (collapsed) classifier accuracy for Experiment $1 \ldots \ldots \ldots \ldots \ldots \ldots . . .20$

2. Pre-stimulus (uncollapsed) classifier accuracy for Experiment $1 \ldots \ldots \ldots \ldots \ldots . .21$

3. Topographic maps of pre-stimulus feature importance for Experiment 1 23

4. Electrode-wise pre-stimulus classifier accuracy for Experiment 1 .

5. Time-frequency-wise pre-stimulus classifier accuracy for Experiment

1

6. Post-stimulus classifier accuracy for Experiment

1 29

7. Topographic maps of post-stimulus feature importance for Experiment 1 . 30

8. Electrode-wise post-stimulus classifier accuracy for Experiment 1. 32

9. Time-frequency-wise post-stimulus classifier accuracy for Experiment

1 33

10. Behavioral Measures for Experiment 2 .45

11. Pre-stimulus (collapsed) encoding-based classifier accuracy for

Experiment 2 .

12. Pre-stimulus (uncollapsed) encoding-based classifier accuracy for

Experiment 2 
13. Topographic maps of encoding-based feature importance for Experiment 2.

14. Pre-stimulus (collapsed) encoding-based classifier accuracy by test

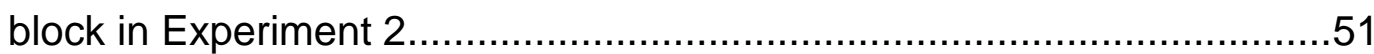

15. Slopes of encoding-based pre-stimulus classifier accuracy across test

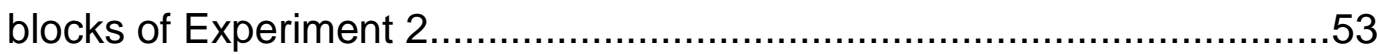

16. Pre-stimulus (uncollapsed) encoding-based classifier accuracy by test

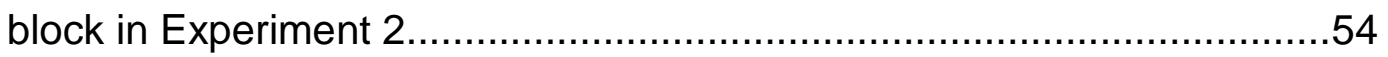

17. Pre-stimulus retrieval-based classifier accuracy for Experiment

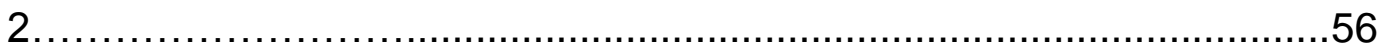

18. Pre-stimulus (collapsed) retrieval-based classifier results by test

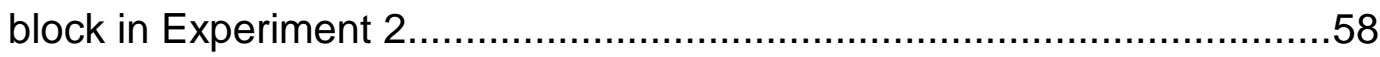

19. Pre-stimulus (uncollapsed) retrieval-based classifier accuracy by test

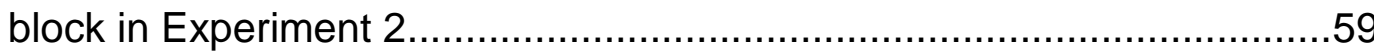




\begin{abstract}
Successful retrieval of episodic memories has been shown to depend on the overlap between the processes engaged during encoding and those re-engaged during retrieval. The ability to strategically adapt cue processing to maximize this overlap, sometimes referred to as "retrieval orientation", has been supported by numerous studies employing electroencephalography (EEG), which demonstrate differences according to the class of memories being sought. However, research in this domain has largely focused on event-related potential (ERP) effects of sustained amplitude that occur after the onset of retrieval cues, thereby failing to indicate whether such effects might be engaged in a preparatory manner before cue onset. Here, we describe two experiments that addressed this issue by employing an analysis approach focusing on the pre-stimulus period of retrieval test trials and capitalizing on the increased sensitivity of multivariate pattern analysis (MVPA) of oscillatory activity. Experiment 1 established evidence of a preparatory form of retrieval orienting and examined the potential fluctuation versus stability of such processing. Experiment 2 attempted to directly modulate the engagement of orienting processes by parametrically manipulating the extent to which responses during the retrieval task are speeded. By novelly quantifying the relationship between multivariate neural correlates of retrieval orientation and behavioral measures of performance, and testing how orienting might be flexibly adjusted to meet task demands, the current project serves as a starting point for developing techniques aimed at improving episodic memory retrieval through strategic control.
\end{abstract}




\section{GENERAL INTRODUCTION}

Episodic memory is traditionally defined in terms of the relationship between a retrieval cue and an existing memory trace. This relationship depends not only on the quality of the trace and the effectiveness of the cue but also on the strategically controlled processes that might maximize the cue-trace interaction at the time of retrieval. Seminal work from experimental psychology demonstrated that the likelihood of retrieval increases with the degree of overlap between the processing of a cue and the representations stored in memory (Tulving \& Thompson, 1973; Tulving, 1983). Furthermore, this overlap is not limited to physical characteristics inherent to the stimuli, but can also involve contextual information present during both encoding and retrieval (Eich et al.,1975) as well as the cognitive operations engaged at both time points (Morris, Bransford, \& Franks, 1977; Jacoby et al., 2005; Rugg et al., 2008). While research on improving episodic memory has focused predominantly on the operations engaged during encoding, which could thereby enhance the strength or durability of the traces formed (Uncapher \& Rugg, 2005; Rebok et al., 2014), much less work has been directed at understanding how cue processing can be oriented strategically to maximize successful retrieval.

Early work on differentiating the operations involved in retrieval cue processing followed the logic of Tulving's 'episodic-semantic distinction' (Tulving, 1983). In order for a retrieval cue to be processed in an episodic manner, the individual must be in a sustained cognitive state, or 'retrieval mode', which increases the 
likelihood of trace-cue overlap and prioritizes episodic information leading to the recreation of the event. Accordingly, behavioral and neural evidence for retrieval mode should be obtained by contrasting an episodic retrieval task with that requiring the retrieval of semantic information. In an initial study that employed event-related potentials (ERPs), Duzel et al., (1999) demonstrated that episodic retrieval was associated with an enhanced positivity at right-frontal electrode sites in comparison to semantic retrieval, and that this distinction slowly evolved over the course of multiple test trials. The sustained time course of the effects was particularly relevant for further investigation of other processes contributing to retrieval (Rugg \& Wilding, 2000), particularly in a manner that depended on the specific nature of episodic retrieval task demands, which is the focus here.

Several studies that have investigated changes in retrieval cue processing, or 'retrieval orientation', in service of accessing different types of episodic information have largely relied on the use of ERPs (Ranganath \& Paller, 1999; Herron \& Wilding, 2004; Werkle-Bergner et al., 2005; Bridger \& Mecklinger, 2012; Rosburg et al., 2014). In one early study, Robb and Rugg (2002) had subjects encode a series of everyday objects that were presented either in picture form or by their corresponding names (words). Recognition memory for the objects was then tested by targeting the different forms in separate blocks, such that subjects were explicitly instructed to respond whether an item was encoded in a particular form or was new (not presented earlier). Analysis of the ERPs obtained during the test phase was restricted to correctly-rejected new test items in order to detect differences in how cue processing was oriented while 
minimizing any potential differences in successful retrieval or post-retrieval processing (Wilding \& Rugg, 1996; Wilding, 1999; Friedman \& Johnson, 2000; also see Rugg \& Wilding, 2000). The new-item ERPs from the word retrieval blocks were more positive-going than those from the picture blocks, and these differences were broadly evident over frontal, central, and posterior electrode sites. Moreover, the effects onset by about 300-ms after stimulus onset and lasted until the end of the recording epoch, suggesting that they reflected the adoption of a sustained cognitive set rather than merely the transient processes elicited by the cue.

Johnson and Rugg (2006b) further investigated the utility of retrieval orientation in a subsequent study by testing whether such processes could be adapted according to the demands of the retrieval task. Subjects in their study completed multiple cycles of encoding pictures and words and then being tested separately on each form of item. The key manipulation involved the nature of the memory test, which employed either a recognition procedure (as described above for Robb \& Rugg, 2002) or an exclusion procedure (Jacoby, 1991). The exclusion procedure requires that items from the targeted form be accepted while new test items are rejected, as in recognition, but it additionally requires that items encoded in the alternative, 'non-targeted', form also be rejected. Thus, the two procedures vary in the specificity with which memories must be retrieved. Consistent with the findings of Robb and Rugg (2002), new-item ERPs were more positive when words as opposed to pictures were targeted for retrieval, and these differences were evident during both the recognition and exclusion tests 
(also see Herron \& Rugg, 2003). Importantly, the effects were larger in magnitude and more sustained in the exclusion test relative to those in the recognition test, suggesting that subjects were more likely to engage in differential cue processing when non-targets had to be rejected. These results thus highlight the potential for subjects to strategically adjust their adoption of retrieval orienting processes in service of task demands.

Whereas the aforementioned findings indicated that retrieval orientation is, to a degree, under the control of subjects, the extent to which orienting could flexibly be adjusted from moment to moment remained unclear. In a series of studies, Herron and colleagues (Herron \& Wilding, 2004; 2006; 2016) tested the limits of orienting retrieval processes on a trial-by-trial basis within a memory test. Their subjects first encoded visually-presented words in the context of two crossed manipulations: to the left or right of a central fixation and by making either an animacy or pleasantness judgment. During a later recognition test, an instructional cue preceded each test item to indicate whether the item's location at encoding or its associated task should be retrieved. Crucially, these cues were administered either in a blocked manner in which the same retrieval dimension was cued over several successive trials (as in the previous studies) or when pairs of the same cue alternated. The new-item ERPs in the blocked tests differed according to the type of cue, suggesting that subjects were able to adopt distinct orienting processes. By contrast, no differences were evident in the ERPs from alternating cue blocks, even when focusing the analysis on trials where the cue was the same as on the previous trial. These findings indicated that, if 
orienting processes can be flexibly engaged, more than two consecutive trials with the same retrieval demands are necessary for it to build up.

Before turning to the specific motivation for the current experiments, the broader implications of understanding and potentially training effective retrieval strategies is addressed. Given the increasing proportion of older adults in the world and the typical association of this population with declining episodic memory function, interventions that might be able to alleviate these memory difficulties are particularly desirable. An obvious target of such intervention are the controlled processes that support memory. Consistent with this idea, Kirchhoff et al., (2012) conducted a functional magnetic resonance imaging (fMRI) study in which older adults were trained to use more effective encoding strategies. Increases in the self-reported use of the trained strategies were correlated with increased activity in several brain regions, including dorsomedial prefrontal cortex and left lateral temporal cortex, and corresponded to behavioral improvement in subsequent memory. In another study, Engvig et al., (2012) trained subjects on a classical encoding strategy known as the method of loci and observed changes in white matter structure in prefrontal cortex that were associated with improvements in memory performance. While the aforementioned findings provide evidence that training is related to structural and functional changes in the brain, it is noteworthy that both studies focus on encoding interventions (also see Rebok et al., 2014). A novel advance of the current project is the focus on modulating retrieval processing, which is further discussed below. 
While the ERP studies discussed earlier have provided consistent evidence that subjects can differentially engage retrieval processes, there are two shortcomings common to each of their analysis approaches that may have limited the detection of robust orientation effects. One issue concerns the sole reliance on effects that occur after the onset of test items (i.e. post-stimulus effects). A potentially stronger test of orienting processes being deployed in a strategic manner would come from investigating neural activity prior to item onset. Such pre-stimulus differences could identify a purer form of information about the deployment of preparatory strategies intended to bias initial cue processing, as opposed to being contaminated by any processes engaged by the cue itself. This approach of focusing analysis on pre-stimulus activity has been shown fruitful in the study of preparatory processes engaged generally during episodic memory encoding (e.g., Otten et al., 2006; Gruber et al., 2013) and retrieval (e.g., Addante et al., 2011), but it has yet to be employed to investigate retrieval orientation. The other issue common to prior studies is their exclusive use of ERP amplitude analyses. Such analyses require that the waveforms are baseline-corrected, typically relative to the pre-stimulus amplitude, which could hinder the detection of effects that are relatively slow to develop and sustained. One way to avoid this issue is to switch to analyzing EEG oscillatory power in one or multiple frequency bands - an approach that has been successful in understanding other aspects of episodic memory (for reviews, see Klimesch et al., 1994; Fell \& Axmacher, 2011; Hanslmayr \& Staudigl, 2014). 
Investigation of the oscillatory dynamics of EEG associated with cognitive processes have a long history dating back to the initial observations of Hans Berger in the 1920s. Modern research on oscillatory activity related to memory have largely focused on four well-established frequency bands: theta $(4-8 \mathrm{~Hz})$, alpha, $(9-12 \mathrm{~Hz})$, beta $(13-30 \mathrm{~Hz})$, and gamma $(31-50 \mathrm{~Hz}$; for reviews, see Klimesch, 1994, 1999; Nyhus \& Curran, 2010). Synchronization in neural firing (i.e. postsynaptic potentials) can lead to increase in power for a particular frequency band, while reductions in power are observed when neural firing is diminished or asynchronous (Salinas \& Sejnowski, 2001; Fries, 2005). Both patterns have been observed in relation to a variety of processes involved in memory. For instance, during encoding, decreases in power in the alpha and beta bands at prefrontal and occipital electrodes has been shown to correlate with subsequent memory performance at retrieval (Long et al., 2014). This finding is consistent with the idea that occipital alpha reflects the inhibition of local neuronal populations that in turn allows information to be maintained in working memory (Nenert et al.2012). Likewise, decreases in beta power have also been demonstrated at the time of memory retrieval (Hanslmayr et al., 2009). Salari and Rose (2016) further assessed the causal role of beta power on recognition memory by using it to trigger test-item presentation. When items were presented immediately following a decrease in beta power, they were more likely to elicit a successful retrieval response relative to items following high beta power states. 
Oscillatory activity in the higher frequency ranges, such as in the gamma band, have additionally been implicated specifically in episodic memory. A recent study conducted on a clinical sample that exhibited severely deficient autobiographical memory showed that gamma synchronization was largely absent in the population (Fuentemilla et al., 2018). By contrast, increases in gamma power were associated with the recovery of rich, integrated information in a control sample of healthy subjects. Multiple studies have also demonstrated that the correlated oscillatory activity across the theta and gamma bands at the time of encoding is also conducive to subsequent retrieval (e.g., Lisman \& Jenson, 2013; Hanslmayr et al., 2016). Such correlations have also been observed with intracranial EEG recordings (Long et al., 2014; Sederberg et al., 2007; Lega et al., 2016) and are theorized to reflect the neural coupling across cortical regions that is indicative of binding mnemonic information.

Here, two experiments are described that investigate and attempt to directly modulate the potential preparatory nature of orienting retrieval processing. Whereas the motivations and specific details for each experiment are described in turn under their corresponding sub-headings (sections 3.1 and 4.1), both experiments address the issues described above by focusing analyses on oscillatory activity obtained during the pre-stimulus period of retrieval test trials. Briefly, Experiment 1 provides novel evidence from multivariate pattern analysis (MVPA; for reviews, see Haynes \& Rees, 2005; Norman et al., 2006) that the patterns of oscillatory activity during this period carry information about the type of memory traces subjects are targeting for retrieval. Experiment 2 then uses a 
similar analysis approach in the context of systematically changing the task demands of the retrieval test. This manipulation is expected to place greater incentive on processing retrieval cues in a manner that maximizes their overlap with existing traces, with the ultimate goal of beginning to understand how subjects might be trained to employ more effective retrieval orienting strategies. 


\section{EXPERIMENT 1}

In Experiment 1, we sought to elucidate the relationship between targeted memory retrieval and pre-stimulus neural activity. As described above, targeting the material form of encoded information (e.g., pictures versus words) has been shown to produce robust ERP differences at that time of retrieval, indicative of the differential orienting of cue processing (e.g., Robb \& Rugg, 2002; Johnson \& Rugg, 2006). In the current experiment, we thus manipulated whether items were encoded as pictures or words. After encoding a mixture of such items, subjects completed multiple memory tests that each targeted items of one encoding form. Regardless of the targeted form, all test items were presented as words in order to hold their physical appearance constant across the different conditions. Each test utilized an exclusion procedure (Jacoby, 1991), which has previously been shown to enhance the magnitude of retrieval orientation effects over a simple recognition procedure (Johnson \& Rugg, 2006). In an example of a targeted test, items encoded as pictures required a positive response, while items encoded as words, along with new (non-studied) items, required a negative response. Analysis of the EEG data according to the targeted material from encoding was then used to identify retrieval orientation differences.

From a theoretical viewpoint, if subjects are differentially engaging orienting strategies to affect the processing of retrieval cues, these effects should be evident prior to established neural correlates of retrieval success, such as the left parietal recollection effect (for review, see Rugg \& Curran, 2007) and the midfrontal negative effect indicative of item familiarity (Strozak, Abedzadeh, \& 
Curran, 2016). Moreover, if such effects are engaged in a preparatory manner, they should be observed even prior to the onset of a retrieval cue. As described earlier, the ability to detect such orienting differences was maximized by focusing analyses on oscillatory activity, which can be assessed in the absence of baseline-correction (cf. ERP amplitude).

In addition to analyzing oscillatory activity in the EEG, MVPA was used in attempt to detect more sensitively any differences between the targeted test conditions and to allow the nature of retrieval orienting processes to vary across subjects. While the temporal resolution of EEG makes it possible to detect the time course of retrieval orientation effects, the spatial resolution leaves much to be desired when attempting to dissociate the effects from multiple underlying neural generators. Additionally, subtle differences in neuroanatomy or neural organization may obscure the reliability of subject-specific effects when combined at the group level. MVPA allows for the scalp topography of EEG effects to vary across subjects, and these effects can then be consolidated into simple descriptive measures that can be tested at the group level (regardless of topography). Because these effects likely engage a distributed set of cortical regions, MVPA is well suited to accurately discriminate between retrieval processes that prioritize the retrieval of specific information and quantify the relationship between retrieval orientation and memory performance. 


\subsection{METHODS}

\subsubsection{Subjects}

Forty-seven undergraduate students from the University of Missouri (MU) participated for partial course credit. All subjects were right-handed, nativeEnglish speakers who had normal or corrected vision and no history of neurological disorders. Informed consent was obtained in accordance with the MU Institutional Review Board (IRB). Of the initial sample, nine subjects were excluded from analysis due to inadequate behavioral performance, and seven additional subjects were excluded due to equipment errors or excessive artifact in the EEG data, leaving thirty-one subjects that were included in all analyses.

\subsubsection{Stimuli}

A pool of 400 pictures of common objects and their corresponding names was used (also see Robb \& Rugg, 2002; Johnson \& Rugg, 2006; Duverne, Motamedinia, \& Rugg, 2009). For each subject, equal proportions of items were randomly assigned to four conditions: encoded as a picture, encoded as a word, presented as a new item during the targeted test phase, or presented as a new item during the final recognition test. All items were presented in word form during the test phases. Words were presented in uppercase white Arial font, with the longest word being approximately $10 \times 1 \mathrm{~cm}$. Each picture was approximately $5 \times 5.5 \mathrm{~cm}$ in size, with the background area of the picture being gray. All stimuli were presented centrally against a black background on a 24-inch widescreen LCD monitor that was cropped to $1024 \times 768$ resolution and viewed at a distance 
of approximately 1 meter. Stimulus presentation was controlled by the Cogent 2000 toolbox (http://www.vislab.ucl.ac.uk) in MATLAB (The MathWorks, Natick, $M A)$.

\subsubsection{Procedure}

Prior to the start of the experiment, subjects were fitted with an electrode cap (lasting about 30 minutes) and given instructions about the encoding phase. After the encoding phase, subjects completed four blocks of a targeted memory test in which retrieval was directed toward items from a particular encoding condition (i.e. picture or word) at a given time. Lastly, a final recognition memory test was administered. Instructions for each memory test were withheld until immediately prior to its start to prevent subjects from adopting any undesirable strategies on the previous phases. Brief practice sessions were administered before each phase to ensure understanding.

During the encoding phase, subjects were presented with an intermixed series of 100 words and 100 pictures. Each item was presented for 1500 ms, preceded by a central white fixation cross for $1000 \mathrm{~ms}$, and followed by a central white fixation cross for a randomly jittered interval between 1500 and 1750 ms. Subjects were instructed to judge whether each item was smaller or larger than a standard shoebox by respectively pressing the " $n$ " and " $m$ " keys on the keyboard with their right index and middle fingers.

Subjects next completed the targeted memory test phase, which was divided into four blocks. Each block employed an exclusion procedure (Jacoby, 1991) to 
target items encoded in a particular form (i.e. pictures or words). Targeted items required a response by pressing the " $m$ " key, and all other items - whether they were from the other form or new - required a response with the "n" key. To control the physical form of test items presented across blocks, all items were presented as words (also see Robb \& Rugg, 2002; Herron \& Wilding, 2004; Dzulkifli \& Wilding, 2005; Johnson \& Rugg, 2006). Speed and accuracy of responding were equally emphasized in the instructions. Each block consisted of 25 targeted items, 25 encoded items from the non-targeted form, and 25 new items. Test items were displayed for $1500 \mathrm{~ms}$, preceded by a central red fixation cross for $1500 \mathrm{~ms}$, and followed by a central white fixation cross for a random interval between 2000 and 4000 ms. The targeted form followed an ABBA sequence across the four test blocks, with the starting form alternating across subjects.

Upon completion of the targeted test blocks, a final recognition memory test was administered. The test employed what is sometimes referred to as the "memory for foils" procedure (e.g., Jacoby et al., 2005), in which subjects had to distinguish between items presented as new during the previous test blocks and items that were entirely new to the experiment (100 of each type). The "n" and " $m$ " keys were respectively used for these responses, with speed and accuracy equally emphasized. The trial timing of the previous test blocks - $1500 \mathrm{~ms}$ for the red fixation cross, $1500 \mathrm{~ms}$ for the test item, and $2000-4000 \mathrm{~ms}$ for the jittered white fixation cross - was again used here. 


\subsubsection{EEG Acquisition and Processing}

EEG data were acquired during all phases of the experiment from $59 \mathrm{Ag} / \mathrm{AgCl}$ electrodes connected to a BrainAmp Standard system (Brain Vision LLC; Durham, NC; http://www.brainvision.com). Electrodes were embedded in an elastic cap (Easycap, Herrsching, Germany; http://www.easycap.de) at the following locations of the International 10-20 system: $\mathrm{Fpz} / 1 / 2, \mathrm{AFz} / 3 / 4 / 7 / 8$, Fz/1/2/3/4/5/6/7/8, FC1/2/3/4/5/6, FT7/8, Cz/1/2/3/4/5/6, T7/8, CPz/1/2/3/4/5/6, TP7/8, Pz/1/2/3/4/5/6/7/8, POz/3/4/7/8, and O1/2. Data were recorded in reference to an electrode placed at FCz, and a ground was located at FT10. Additional electrodes were adhered to the mastoids (M1 and M2), and vertical and horizontal EOG were recorded from electrodes positioned on the outer canthi (LO1 and LO2) and below the left eye (IO1). Electrode impedances below $5 \mathrm{k} \Omega$ were established prior to the start of the experiment. Data were recorded at a sampling rate of $1 \mathrm{kHz}$ and with an amplifier bandwidth of $.01-100 \mathrm{~Hz}$.

Offline processing of the EEG data was implemented with the EEGLAB toolbox (Delorme and Makeig, 2004; http://sccn.ucsd.edu/eeglab) in MATLAB. The continuous data were band-pass filtered from $.05 \mathrm{~Hz}$ to $50 \mathrm{~Hz}$, down-sampled to $200 \mathrm{~Hz}$, re-referenced to the mastoid average, and epoched from -3000 ms to 2000 ms relative to stimulus (word) onset. The extended pre-stimulus period of the epoch allowed us to assess the cue (red fixation cross) portion of the trial that was of central focus to our hypotheses. Independent components analysis (ICA) was used to identify artifacts in the data based on scalp topography and spectral composition (Jung et al., 2000). The SASICA toolbox (Chaumon, Bishop, \& 
Busch, 2015; https://github.com/dnacombo/SASICA) was additionally used to guide the selection of artifactual components, which were then manually rejected. Finally, the epoched data were time-frequency transformed using a Morlet wavelet decomposition, with linearly spaced frequencies from 4 to $50 \mathrm{~Hz}$.

\subsubsection{EEG Analysis}

Analyses of the EEG data utilized the Princeton Multi-Voxel Pattern Analysis toolbox (The Princeton Neuroscience Institute, Princeton, NJ;

https://github.com/PrincetonUniversity/princeton-mvpa-toolbox) in MATLAB. Each 100-ms time bin across the recording epoch comprised a pattern of all of the electrode $\times$ frequency band combinations $(59 \times 47=2773$ features $)$. After $z$ scoring each feature separately across all test blocks, the patterns were submitted to L2-regularized logistic regression classifiers that were trained for each subject to detect differences between the targeted forms of test items. A cross-validation procedure was employed in which a classifier was trained on two test blocks and evaluated on the remaining two blocks, and this procedure was then repeated by switching the training and evaluation sets. Classifier accuracy was used as a metric of performance, with chance performance being .5 (given the two stimulus classes) and accuracy above chance indicating that orienting occurred for the targeted class of encoding items. 


\subsection{RESULTS}

\subsubsection{Behavior}

The proportions of correct responses and associated response times (RTs) for the targeted memory tests are summarized in Table 1. These measures were analyzed with separate ANOVAs employing factors of targeted material (picture or word) and item type (target, nontarget, or new). The analysis of proportions gave rise only to an interaction between the two factors $\left(F_{1.4,41.4}=9.74, P=.001\right.$; degrees of freedom are Greenhouse-Geisser adjusted when necessary). Followup contrasts of targeted material for each item type revealed that correct response proportions to targets were higher when words compared to pictures were targeted $\left(t_{30}=2.71, p=.011\right)$, but the opposite pattern was evident for nontargets $\left(\mathrm{t}_{30}=3.24, \mathrm{p}=.003\right)$ and new items $\left(\mathrm{t}_{30}=2.53, \mathrm{p}=.017\right)$. The ANOVA of correct RTs revealed no significant main effects or interaction (all ps > $.3)$.

\section{Table 1. Behavioral measures for Experiment 1}

Mean (and SEM) proportions of correct responses and associated response times (RTs; in milliseconds) from the targeted memory test of Experiment 1

\begin{tabular}{|l|l|l|l|}
\hline Targeted material & Item type & Proportion correct & Correct RT \\
\hline Picture & Target & $.70(.02)$ & $1124(55)$ \\
\hline & Nontarget & $.84(.02)$ & $1137(52)$ \\
\hline & New & $.80(.02)$ & $1130(52)$ \\
\hline Word & Target & $.77(.02)$ & $1147(54)$ \\
\hline & Nontarget & $.76(.02)$ & $1131(53)$ \\
\hline & New & $.75(.03)$ & $1122(55)$ \\
\hline
\end{tabular}


For the final recognition test, subjects had to distinguish between items that previously served as new items during the targeted test blocks and those that were entirely new to the experiment. The mean proportions of correct responses for items from the picture and word targeted blocks were both .82 (SEMs $=.03$ and .02, respectively), whereas the mean correct proportion for new items was $.78(\mathrm{SEM}=.02)$. The respective correct RT means (and SEMs) were 1261 (73), 1285 (78), and 1261 (73) ms. ANOVAs employing a single factor of item type (from the targeted picture block, from the targeted word block, or entirely new) revealed no significant effects for the correct proportions $(F<1)$ or $R T s\left(F_{1,30}=\right.$ $3.4, p=.076)$. There was thus no indication that distinct orienting during the targeted memory test carried over to subsequent behavioral differences (cf. Jacoby et al., 2005; Shimizu \& Jacoby, 2005).

\subsubsection{EEG}

\section{Classification of pre-stimulus activity}

To get an initial impression of the ability for EEG from the pre-stimulus period to be classified according to whether studied pictures versus words were targeted, data from the entire 1500-ms period was first averaged for both classifier training and testing. As shown in Figure $1 \mathrm{~A}$, the resulting mean classifier accuracy was $.64(\mathrm{SEM}=.02)$ and above the chance level of .5 (given two classes; $\mathrm{t}_{30}=6.58, \mathrm{p}$ $=1.4 \times 10^{-7}$, one-tailed). Given that the types of test items (target, nontarget, and new) were randomly presented during each block, we also predicted that there 
would be no pre-stimulus differences in classification accuracy according to item type. This pattern was confirmed, as displayed in Figure 1B for each item type. Mean accuracy was again around .64 and above chance in all cases $\left(t_{30}=6.17\right.$, 5.83, and 6.74, $p=4.4 \times 10^{-7}, 1.1 \times 10^{-6}$, and $9.0 \times 10^{-8}$, respectively). An ANOVA across item type indicated no differences $(F<1)$. These findings therefore provide support for robust orienting effects during the pre-stimulus period, which can be tested without restriction to just correctly-rejected new items (cf. Rugg \& Wilding, 2000). 

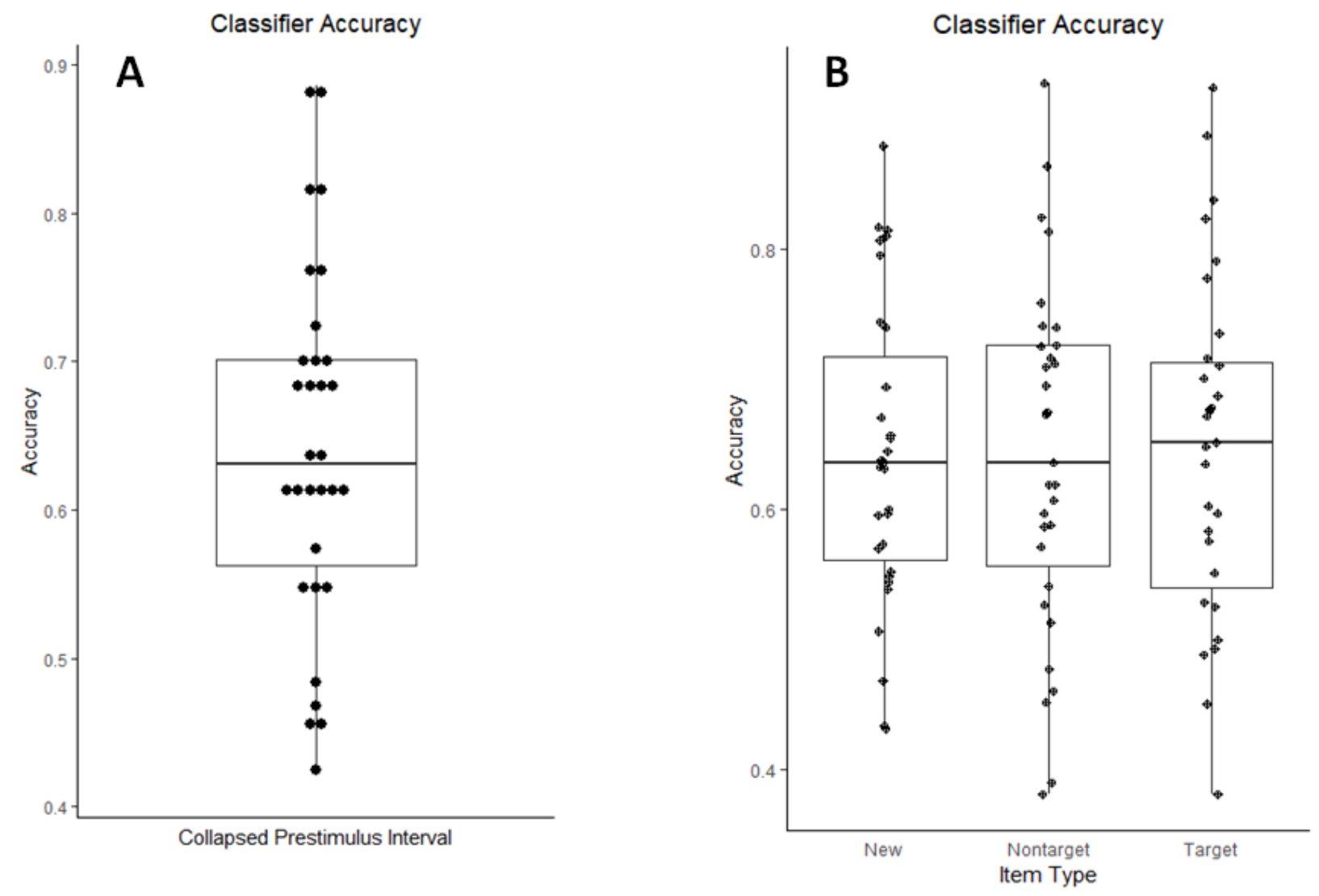

Figure 1. Pre-stimulus (collapsed) classifier accuracy for Experiment 1

A) Classifier accuracy collapsed over the entire 1500-ms pre-stimulus interval and over all item types (target, nontarget, and new) in Experiment 1. The box plot indicates the median, 25- and $75-\%$ intervals, and range of accuracy values. Individual points correspond to the accuracy values for each subject.

B) Collapsed classifier accuracy determined in the same way as in Panel A, but segregated according to item type (target, nontarget, and new).

Next, we examined the time course of pre-stimulus orienting effects by using the classifier trained on data collapsed over the entire 1500-ms period (as above) to evaluate EEG patterns from successive 100-ms time bins across the same period. Figure 2A displays the time courses collapsed across item type. As is apparent from the figure, accuracy was stable around .57 across the all time bins and significantly greater than chance at each bin ( $\mathrm{t}_{30}$ range: 4.63 to $6.18, \mathrm{p}$ range: 
$3.3 \times 10^{-5}$ to $\left.4.3 \times 10^{-7}\right)$. The stability of accuracy was further confirmed with a oneway ANOVA that resulted in a non-significant effect of time bin $(F<1)$.

Segregating the results according to item type also gave rise to stable accuracy around .55 to .60 , as shown in Figure 2B. These measures were again significantly above chance for each item type in each bin ( $t_{30}$ range: 2.88 to 6.26 , p range: $3.6 \times 10^{-3}$ to $\left.3.4 \times 10^{-7}\right)$. A two-way ANOVA of these data, employing factors of item type and time bin, also gave rise to no significant effects (all Fs < 1).
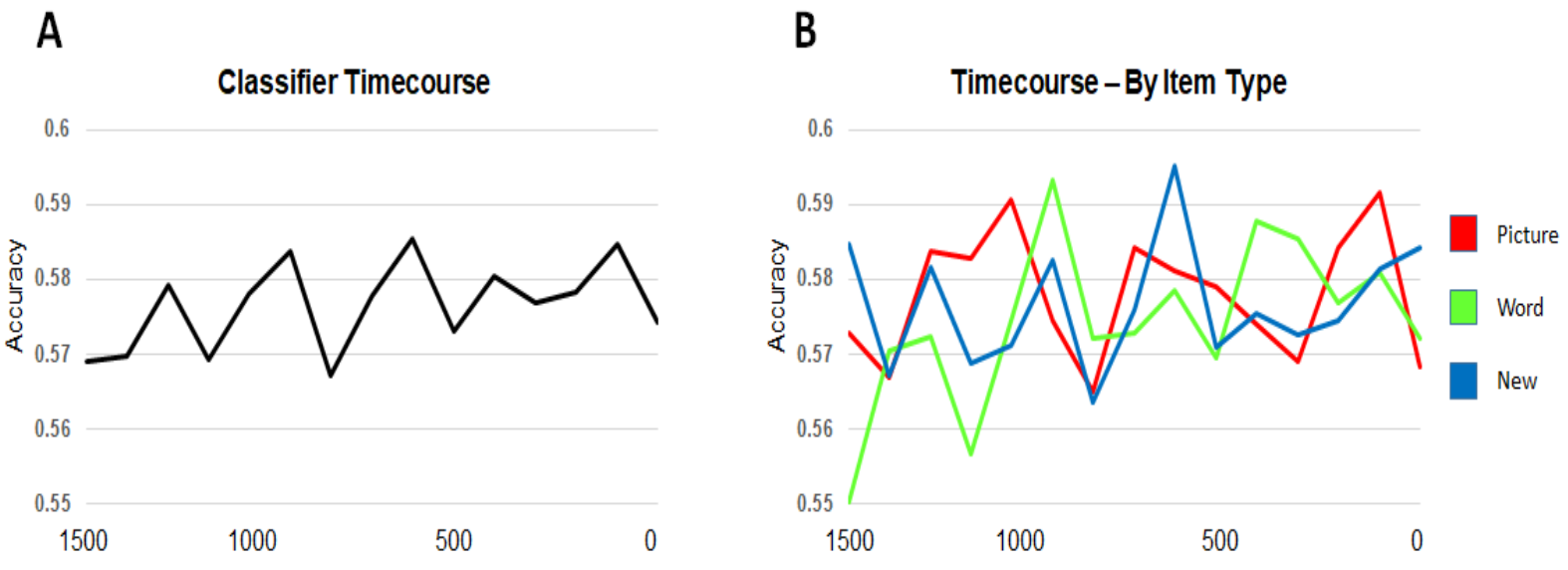

Figure 2. Pre-stimulus (uncollapsed) classifier accuracy for Experiment 1

A) Group mean classifier accuracy by 100 -ms time bins across the pre-stimulus interval, collapsed over all item types (target, nontarget, and new) in Experiment 1.

B) Group mean classifier accuracy determined in the same way as in Panel A, but segregated according to item type (target, nontarget, and new).

To understand which features were important for the classification analysis described above, we extracted the final, trained weights for each feature. Because the classification is binary, the weight of a particular feature for one 
condition (e.g., targeting pictures) just takes the opposite sign for the other condition (e.g., targeting words). These weights were then collapsed over four traditional EEG frequency bands for simplicity: theta $=4-8 \mathrm{~Hz}$, alpha $=9-12 \mathrm{~Hz}$, beta $=13-30 \mathrm{~Hz}$, and gamma $=31-50 \mathrm{~Hz}$ (also see Nyhus \& Curran, 2010; Addante et al., 2011). To highlight the consistency of weight values across subjects, t-values (tested against 0 , where 0 corresponds to "unimportant") were computed at each electrode in each frequency band, and the resulting topographic maps are provided in Figure 3. Note that these maps are to be interpreted purely for descriptive purposes, as each subject's data were trained with an independent classifier and we had no a priori hypotheses about topography. As shown, positive weights for targeting pictures (and negative weights for targeting words) were evident bilaterally over central and temporal sites at lower (theta and alpha) frequencies, and right-lateralized at higher (beta and gamma) frequencies. Additionally, negative weights for targeting pictures (positive for words) were evident over posterior sites in the beta range and more widespread over the scalp in the gamma range. We also created maps by multiplying each trained weight with the respective measure of frequency power for that feature, keeping the products of the signed weights and power separate (as in Polyn et al., 2005; Johnson et al., 2009; McDuff et al., 2009). Those maps were qualitatively similar to the maps of weights alone and corresponding $\mathrm{t}-$ values. 
Theta $(4-8 \mathrm{~Hz})$

Alpha $(9-12 \mathrm{~Hz})$
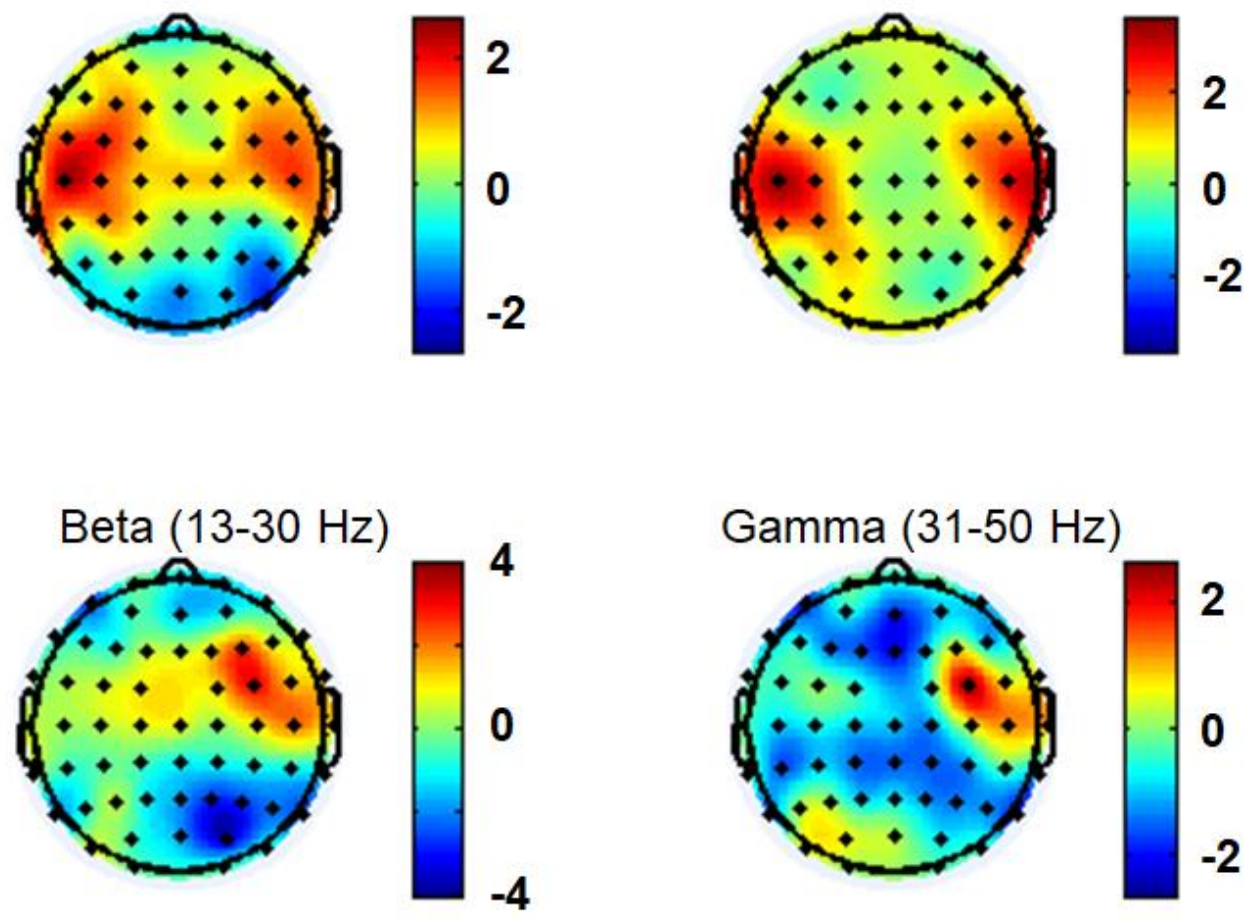

\section{Figure 3. Topographic maps of pre-stimulus feature importance for Experiment 1}

Topographic maps of trained weights assigned to each feature, collapsed into traditional EEG frequency bands, and transformed into t-values (against 0). Warm colors represent positive importance values for targeting pictures (and negative for words), whereas cool colors represent positive importance values for targeting words (and negative for pictures).

To identify converging evidence for the features that were important to the picture versus word classification, we conducted two additional sets of classification analyses, following recent procedures developed by Morton and Polyn (2017). One of these analyses focused on the frequency $\times$ time bin pattern at each electrode, and the other focused on the electrode-wise pattern in each combination of frequency and 100-ms pre-stimulus time bin. By cycling over 
these patterns separately, we can obtain a clearer picture of the electrodes, frequencies, and time bins that carried information distinguishing pictures and words. For the former, time-frequency analysis, patterns of 705 features (47 frequency bands $\times 15$ time bins) were submitted to separate classifiers. The results are shown in Figure 4A, where it is apparent that that accuracy was numerically above chance for the limited data at each electrode (range $=.51$ to .56). Transforming these accuracy measures to t-values, as shown in Figure 4B, gives a better impression of the reliability of effects, despite the limited range of accuracy. Specifically, the maximal effects were evident at electrodes over frontopolar (Fpz: $\left.t_{30}=3.82, p=3.2 \times 10^{-4}\right)$ and left posterior scalp (P5: $t_{30}=3.18, p$ $\left.=.0017 ; \mathrm{PO} 7: \mathrm{t}_{30}=3.28, \mathrm{p}=.0013\right)$. We again note, however, that these statistics should be used primarily for descriptive purpose only. 


\section{Electrode-wise Classification}

A

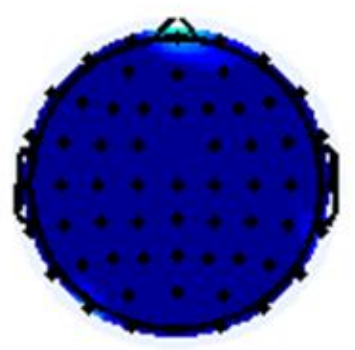

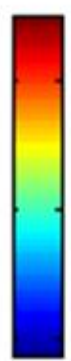

.58

.56

B

.54

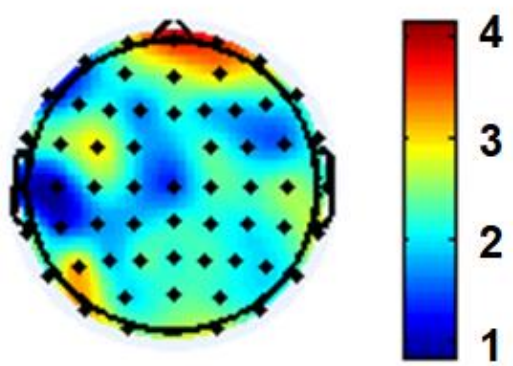

Figure 4. Electrode-wise pre-stimulus classifier accuracy for Experiment 1

A) Topographic map of accuracy based on classifying the time-frequency pattern separately for each electrode. All accuracy values were above the chance level of .5 .

B) Topographic map of accuracy from Panel A, transformed into t-values (against .5). The maximal t-value was at electrode Fpz.

For the second set of classifications to determine feature importance, the electrode-wise patterns were classified for each frequency band $\times$ time bin combination. The results of these analyses are provided in Figure 5. As shown (see Figure $5 A$ ), accuracy ranged from just below chance at .48 to about .60. It is important to keep in mind that the below-chance values may be due to the fact that these classifications are based on only a limited (and potentially noisy) set of 59 features. The transformation of these accuracy measures into t-values (against the chance level of .5; Figure 5B) confirmed the noise that seemed inherent at lower (below $20 \mathrm{~Hz}$ ) frequencies. Shown in Figure 5C are the accuracy results collapsed over frequency bands, which confirmed the 
impression we obtained earlier that classifier performance was stable (at around .54) across 100 -ms time bins during the pre-stimulus interval. Adding to these findings, we also collapsed the classifier accuracies across time bins to examine the effects at different frequency bands. These results, shown in Figure 5D, indicate the increase in accuracy at higher frequencies. Together, these findings thus suggest that the classification of pre-stimulus oscillatory activity picks up on effects that are likely distinct from the low-frequency effects typically highlighted by analyses of post-stimulus ERP amplitude (e.g., Robb \& Rugg, 2002; Herron \& Wilding, 2004; Johnson \& Rugg, 2006b). 
Time-Frequency Classification

Pre-stimulus Period
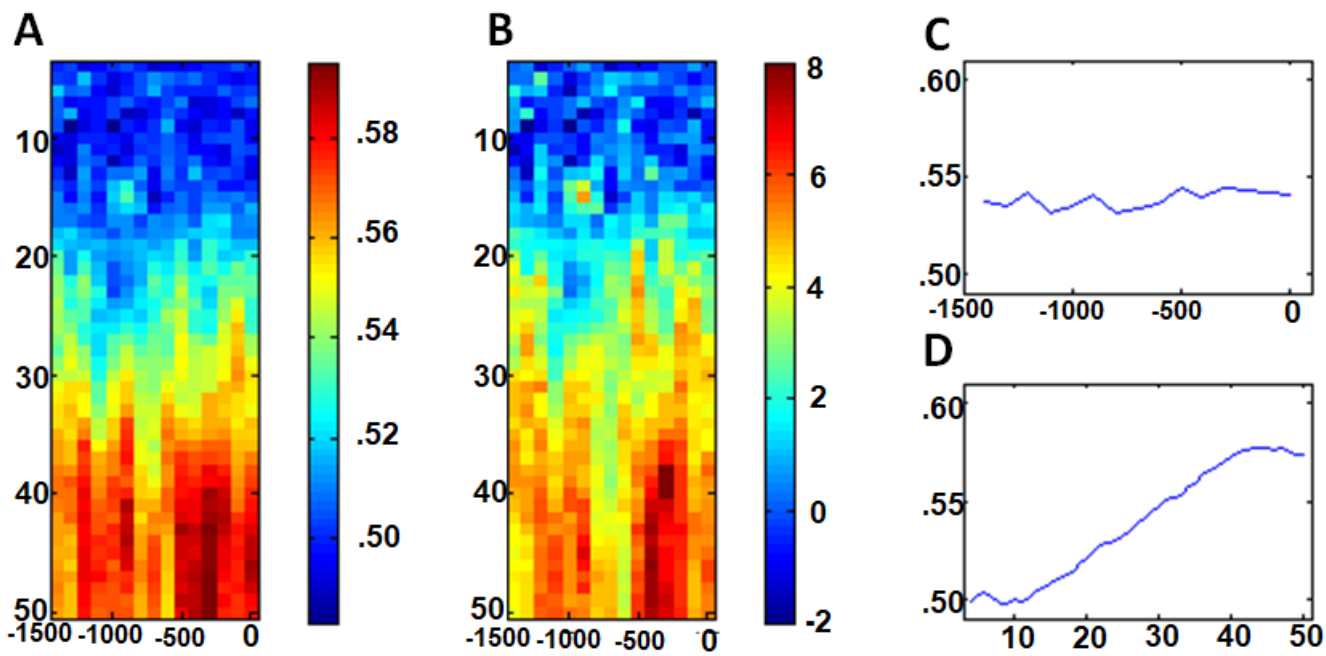

\section{Figure 5. Time-frequency-wise pre-stimulus classifier accuracy for Experiment 1}

A) Classifier accuracy during the pre-stimulus period of Experiment 1, based on classifying each electrode pattern separately for each time bin (-1500 to $0 \mathrm{~ms}$, in 100-ms intervals) and frequency band $(4$ to $50 \mathrm{~Hz}$ ).

B) T-values (against .5) corresponding to classifier accuracy from Panel A.

C) Classifier accuracy from Panel A, collapsed over frequency bands to highlight the time course.

D) Classifier accuracy from Panel A, collapsed over time to highlight the frequency-based differences.

\section{Classification of post-stimulus activity}

We next turned to the EEG data from the post-stimulus period, in order to begin to relate our classification findings to results to previous studies of retrieval orientation that focused on this period. First, we were interested in testing whether there were any qualitative distinctions between pre- and post-stimulus oscillatory activity related to orienting. For these analyses, only the data 
corresponding to correctly-rejected new items were utilized, as targets and nontargets could give rise to successful retrieval (as well as post-retrieval) effects and thereby confound the assessment of orienting (Rugg \& Wilding, 2000). EEG data from the 1500-ms period following test-item onset were first averaged across time and used for classifier training and testing. Figure $6 \mathrm{~A}$ displays the results of this analysis, whereby accuracy was comparable to that from the prestimulus period at a level of $.62(\mathrm{SEM}=.02)$ and significantly greater than chance $\left(t_{30}=5.93, p=8.6 \times 10^{-7}\right.$, one-tailed). Using this trained classifier, we next assessed the time course of accuracy by separately testing it on data from successive 100-ms time bins. As was the case for the pre-stimulus time course, classifier accuracy in the post-stimulus period was stable at around .55 to .60 , as shown in Figure 6B. The accuracy measure for each time bin again significantly above chance ( $t_{30}$ range: 3.10 to 5.84 , p range: .002 to $1.1 \times 10^{-6}$ ) and there was no evidence from a one-way ANOVA for any changes across post-stimulus time $(\mathrm{F}<1)$. 

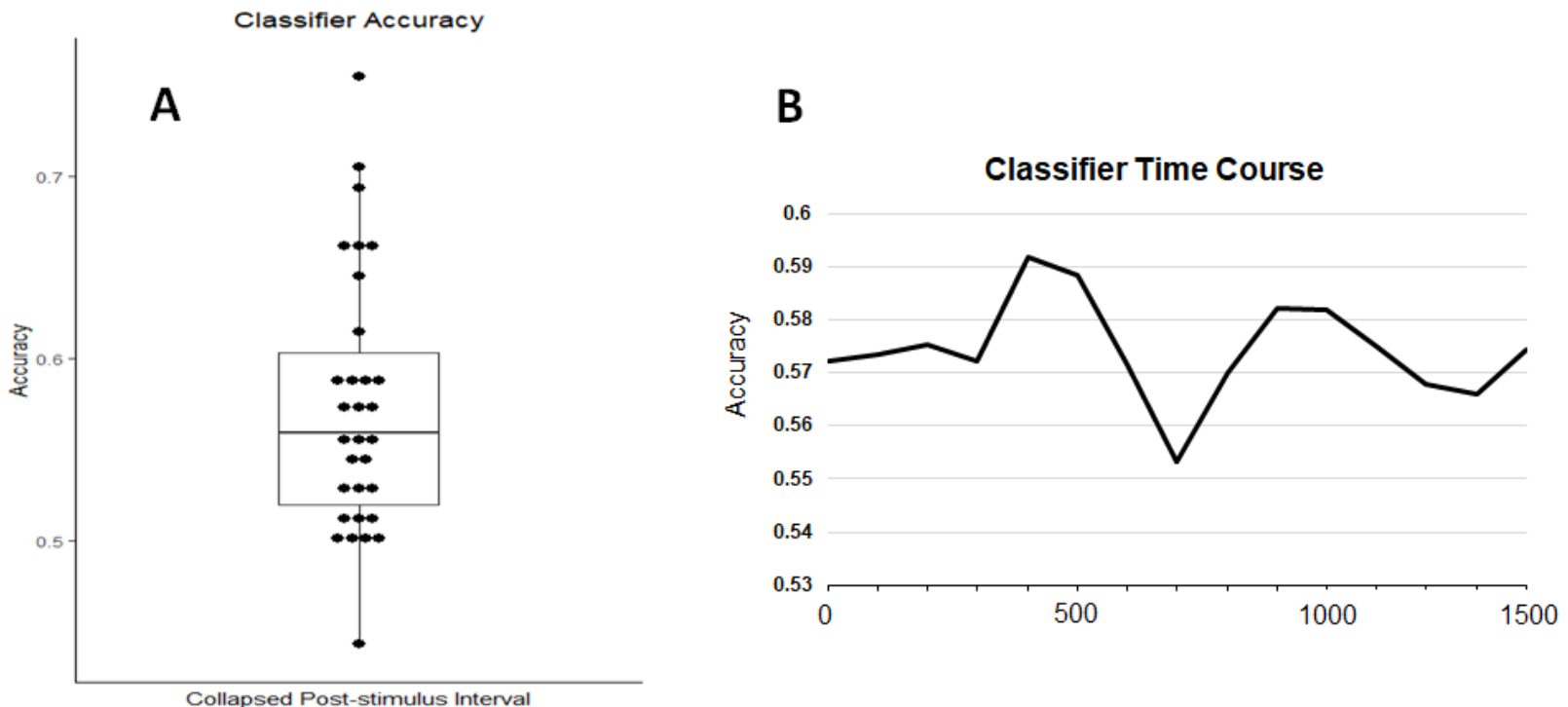

\section{Figure 6. Post-stimulus classifier accuracy for Experiment 1}

A) Classifier accuracy collapsed over the entire 1500-ms post-stimulus interval for correctlyrejected new items in Experiment 1. The box plot indicates the median, 25- and 75-\% intervals, and range of accuracy values. Individual points correspond to the accuracy values for each subject.

B) Group mean classifier accuracy by 100 -ms time bins across the post-stimulus interval for correctly-rejected new items in Experiment 1.

Next, to identify the important features for classifying the post-stimulus data, we extracted the trained weights as was done earlier. The resulting topographic data were again collapsed according to four traditional frequency bands (theta, alpha, beta, and gamma) and transformed into t-values (against a chance value of 0 , i.e. "unimportant). Figure 7 displays the topographic maps of these t-values. As shown, the theta and gamma bands consistently carried positive weights for the blocks that targeted words (negative weights for targeting pictures), and these importance values were widely distributed over the scalp. By contrast, a widespread distribution of positive weights for targeting pictures was evident in 
the alpha band. Finally, importance values in the beta band were positive across frontal scalp for targeting pictures and positive across posterior scalp for targeting words. As was stated earlier, these maps of importance are to be used primarily for descriptive purposes, so we didn't conduct any further statistical tests (beyond the t-transformations) between bands or experiments.

\section{Importance Maps}
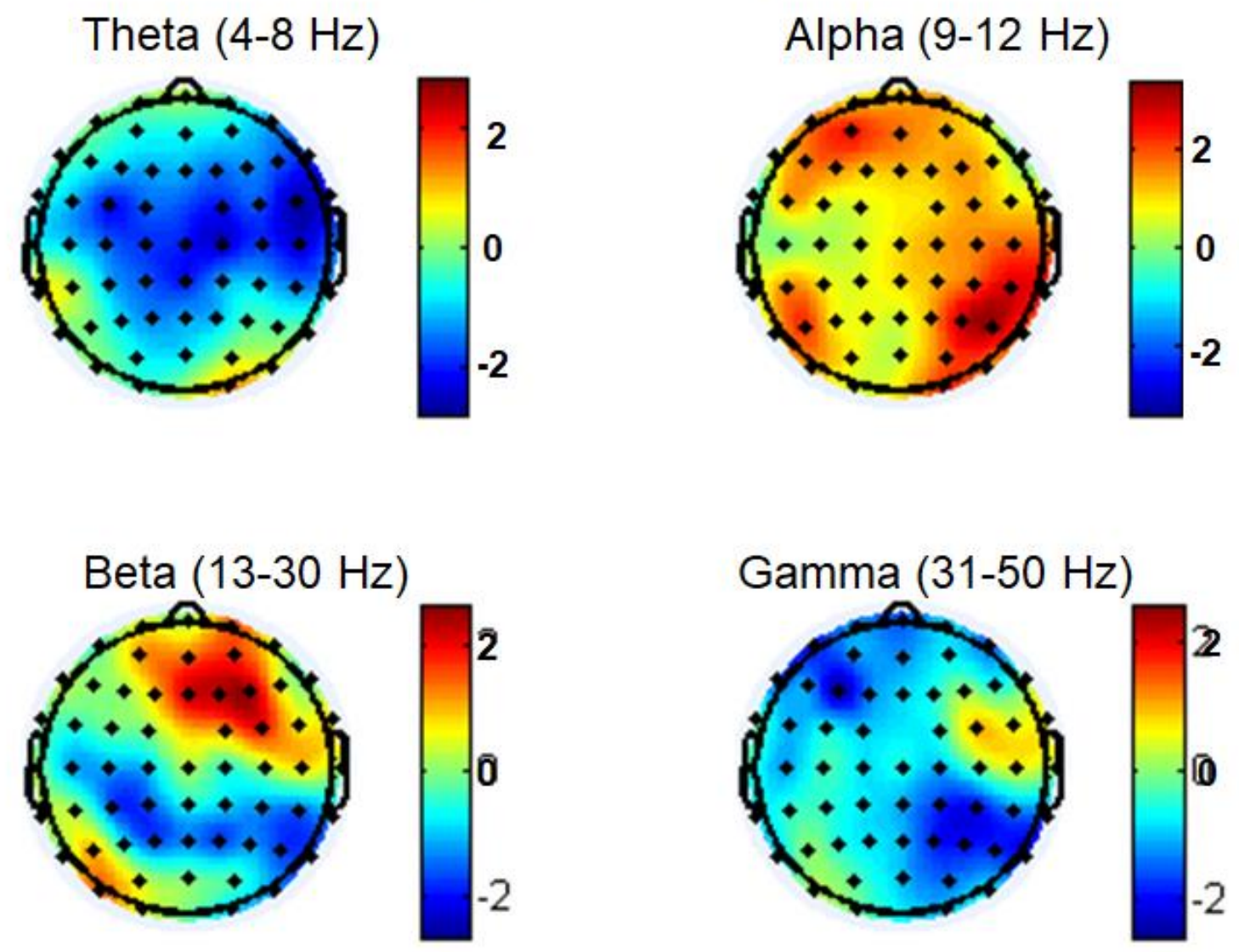

Figure 7. Topographic maps of post-stimulus feature importance for Experiment 1

Topographic maps of trained weights assigned to each feature, collapsed into traditional EEG frequency bands, and transformed into t-values (against 0 ). Warm colors represent positive importance values for targeting pictures (and negative for words), whereas cool colors represent positive importance values for targeting words (and negative for pictures). The classification analysis producing these maps was based only on correctly-rejected new items. 
Further analyses of feature importance were carried out with the separate timefrequency-wise and electrode-wise classifications that were described earlier for the pre-stimulus data (see Figures 4 and 5). Classifying the frequency $\times$ time bin (100-ms) pattern at each electrode revealed that accuracy was numerically above chance at each electrode (range $=.51$ to .56 ), as shown in Figure 8A. Transforming these results into t-values, as displayed in Figure 8B, indicated multiple scalp locations of reliable effects, including over bilateral posterior sites (e.g., $\mathrm{P} 6: \mathrm{t}_{30}=3.62, \mathrm{p}=.001 ; \mathrm{P} 7: \mathrm{t}_{30}=3.80, \mathrm{p}=6.5 \times 10^{-4}$ ), right-lateralized frontal sites (e.g., FC2: $\mathrm{t}_{30}=3.61, \mathrm{p}=.001$ ), and the anterior pole (e.g., Fpz: $\mathrm{t}_{30}=3.60, \mathrm{p}$ $=.001$; as was the case for the pre-stimulus effects). Finally, the electrode-wise patterns at each combination of frequency band and time bin were classified, the results of which are shown in Figure 9. Accuracy across all combinations ranged from .47 to .59 (Figure 9A), with accuracy across time (collapsed over frequency bands) being relatively stable at around .53 (Figure 9C). As is shown in Figure 9D and was the case for the pre-stimulus analyses, classifier accuracy also increased with frequency. Again, this pattern of findings suggests that the classification of post-stimulus oscillatory activity is sensitive to aspects of the EEG that are distinct from the low-frequency effects that likely dominate ERP amplitude. 


\section{Electrode-wise Classification}
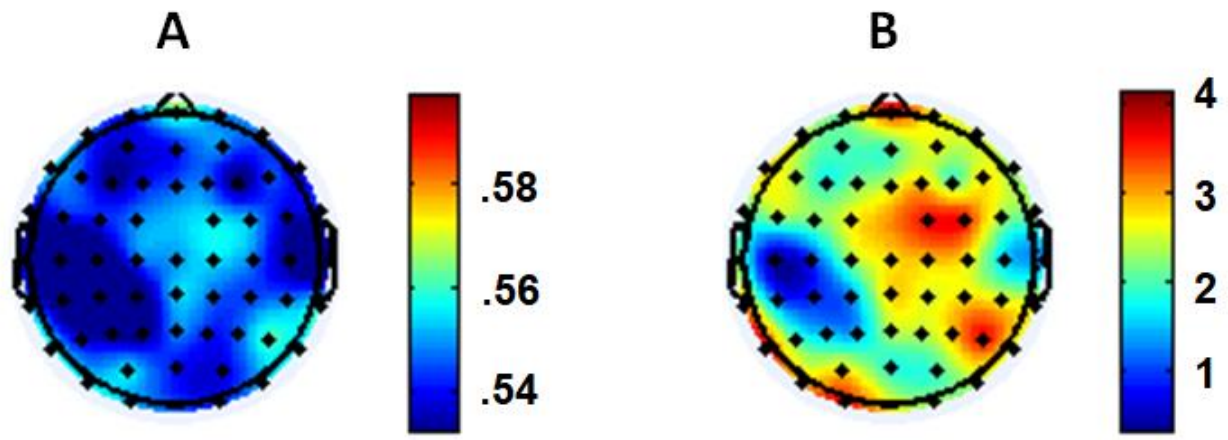

Figure 8. Electrode-wise post-stimulus classifier accuracy for Experiment 1

A) Topographic map of accuracy based on classifying the time-frequency pattern separately for each electrode. All accuracy values were above the chance level of .5.

B) Topographic map of accuracy from Panel A, transformed into t-values (against .5). The maximal t-value was at electrode Fpz. 
Time-Frequency Classification

Post-stimulus Period

A

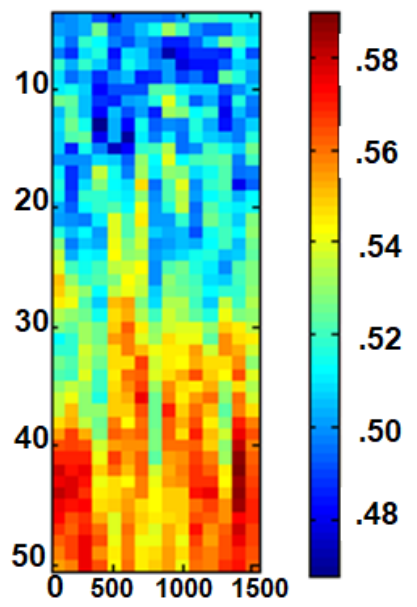

B

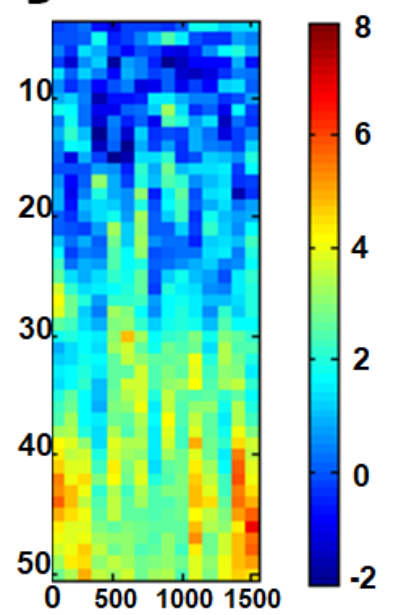

C

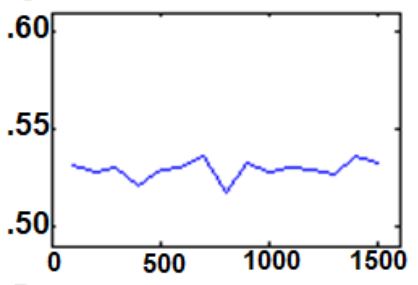

D

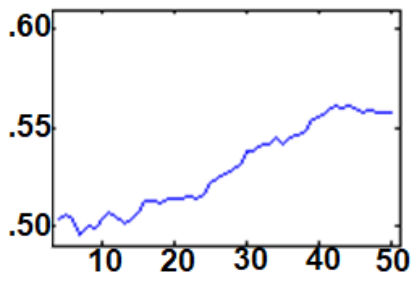

Figure 9. Time-frequency-wise post-stimulus classifier accuracy for Experiment 1

A) Classifier accuracy during the pre-stimulus period of Experiment 1, based on classifying each electrode pattern separately for each time bin (-1500 to $0 \mathrm{~ms}$, in 100-ms intervals) and frequency band $(4$ to $50 \mathrm{~Hz}$ ).

B) T-values (against .5) corresponding to classifier accuracy from Panel A.

C) Classifier accuracy from Panel A, collapsed over frequency bands to highlight the time course.

D) Classifier accuracy from Panel A, collapsed over time to highlight the frequency-based differences.

\subsection{DISCUSSION}

Experiment 1 sought to establish that prior to the presentation of items on a memory test, neural evidence could be obtained that subjects strategically orient retrieval processing toward distinct encoding conditions. To this end, subjects encoded a series of pictures and words and then undertook a memory test in which an exclusion procedure was used to separately focus (target) retrieval on 
one type of encoding material. By examining pre-stimulus oscillatory activity at test, in conjunction with the employment of multivariate pattern analysis (MVPA), we demonstrate several novel forms of support for the notion of strategically orienting retrieval processes. Specifically, above-chance decoding of whether subjects were targeting the picture versus word conditions was present when averaging over the entire (1500-ms) prestimulus interval, and additionally took the form of a sustained time course throughout this interval. Moreover, analogous analyses focused on post-stimulus oscillatory activity revealed similar effects of accurate decoding that was sustained for at least another $1500 \mathrm{~ms}$. Together, these findings highlight the enhanced sensitivity of MVPA for detecting differential forms of retrieval processing, as well as the added benefit of being able to utilize all test items for pre-stimulus analysis as opposed to only correctlyrejected new items for post-stimulus analysis (see Rugg \& Wilding, 2000).

In addition to the consistent evidence of retrieval orienting obtained with MVPA, the comparison of such effects with those from traditional ERP analyses is also striking. As outlined in the Introduction, electrophysiological support for retrieval orientation has come exclusively from the analysis of post-stimulus ERP amplitudes (e.g., Robb \& Rugg, 2002; Herron \& Wilding 2004, 2006; Johnson \& Rugg 2006a, 2006b; for a recent meta-analysis, see Rosburg \& Mecklinger, 2017). Whereas the sustained effects of orienting typically obtained with ERP amplitudes - often lasting 500 ms or longer - suggest that they are driven predominantly by low-frequency activity, the findings obtained here with MVPA 
were strongest at frequency bands of about $20 \mathrm{~Hz}$ or greater. We return to the implications of these differences in the General Discussion. 


\section{EXPERIMENT 2}

Having established evidence from patterns of pre-stimulus oscillatory activity for differential orienting of retrieval processes, we next attempted to further understand the extent to which these operations can be flexibly adapted to the changing demands of retrieval. To meet this goal, Experiment 2 introduced a response-deadline procedure whereby the memory judgment on each trial was encouraged to be made within a set time period. The deadline was set on an individual-subject basis, based on the response times (RTs) during an initial test block that did not include a deadline. Once the deadline was introduced (in the second test block), its latency was gradually shortened across several subsequent blocks in increments of $10 \%$ of the subjects initial median RT. Requiring judgments that were increasingly speeded was predicted to emphasize a greater need for engaging in a effective retrieval orienting strategies in order to maintain accurate memory performance. Following several blocks of shortening the response deadline, two final test blocks were included in which the deadline was gradually relaxed toward the subject's initial median value. We hypothesized that such relaxation would afford the ability to test whether there were lasting effects of practice with more effective orienting or the employment of orienting would become more relaxed.

Aside from the introduction of the response deadline procedure, the general experimental design of Experiment 2 is similar to that of the previous experiment, with two notable exceptions. The first is that, due to the expected increased difficulty of the retrieval phase resulting from the deadlines, we switched from the 
exclusion procedure to an old/new recognition task. This modification was intended not only to ensure that the current subjects could complete the memory task effectively, but also to allow us to move toward a simpler form of memory task that may be beneficial in future studies for testing elderly subjects (also see Duverne, Motamedinia, \& Rugg, 2009). The second exception involved switching to the using a single type of stimulus material during both the encoding and retrieval phases. With this change, the encoding manipulation consisted of viewing words in the context of two different tasks - with one referring to the physical appearance of the denoted object and the other related to its functions (also see Dzukifli \& Wilding, 2005; Bridger \& Mecklinger, 2012; Rosburg, Johansson, \& Mecklinger, 2013; Herron \& Wilding, 2016). This exception was expected to provide the benefit of including data from the encoding phase in the classification analyses; doing so was not possible in Experiment 1, as the classification would have likely focused on the physical differences between pictures and words at encoding which was not present again during the memory test. Other than that change in analysis strategy, MVPA was focused primarily on oscillatory activity from the pre-stimulus period of test trials, as the effects were shown previously to be sustained across trial time and doing so maximized the number of trials that could be included for analysis.

\subsection{METHODS}

\subsubsection{Subjects}


Thirty-four undergraduate students from the University of Missouri (MU) participated for partial course credit. All subjects were right-handed, nativeEnglish speakers who had normal or corrected vision and no history of neurological disorders. Informed consent was obtained in accordance with the MU IRB. Data from three subjects were excluded from analysis due to having low behavioral performance. Two additional subjects were excluded due to terminating the experiment early, leaving a remaining sample of twenty-nine subjects which were included in analyses.

\subsubsection{Stimuli}

A pool of 432 names of common objects was used (also see Experiment 1; Johnson \& Rugg, 2006a, 2006b). A subset of 216 words were randomly selected from the pool and presented during the encoding phase (108 for each encoding task condition, as described below). The encoded words were presented again during the test phase (as 'old' items) along with the remaining 216 words that served as 'new' items. The words were displayed in uppercase white Arial font against a black background, with the longest word being approximately $10 \times 1$ $\mathrm{cm}$. Stimuli were presented centrally on a 24-inch widescreen LCD monitor (cropped to $1024 \times 768$ resolution) at a viewing distance of approximately 1 meter. The Cogent 2000 toolbox for MATLAB was used to control stimulus presentation.

\subsubsection{Procedure}


Upon arrival, informed consent was obtained, subjects were fitted with an electrode cap (lasting approx. 30 minutes), and encoded a list of words under differential task manipulations. Following encoding, subjects completed a series of targeted memory test blocks where response deadline was parametrically manipulated and the target for retrieval randomly alternated. Due to the random order of targeted retrieval blocks, task instructions were withheld until prior to the start of a block. A practice version of the targeted retrieval block was administered prior to the start of the first two retrieval blocks.

The encoding phase consisted of presentation of a series of 216 words. Subjects were to complete one of two tasks for each word. For the 'artist' task, subjects were instructed to rate the difficulty an artist would have drawing the object corresponding to the word, using a four-point scale $(1=$ easy to $4=$ hard). The "function" task required thinking about different uses for the object and then making a response indicating the number of functions generated (from 1 to 4 ). Subjects made the 1 through 4 responses by respectively pressing the "m", ",", ".", and "/" keys on the keyboard with their right index through little fingers. Each item was presented for $1500 \mathrm{~ms}$, preceded by a central white fixation cross for $1000 \mathrm{~ms}$, and followed by a central white fixation cross for a randomly jittered interval between 1500 and 1750 ms. To maximize differences in processing for the two encoding tasks, a given task was used for mini-blocks of six items in a row as opposed to randomly intermixing the tasks. The tasks alternated throughout the encoding phase, with the starting task counterbalanced across subjects. Instructional cues (e.g., 'Do ARTIST task') designating the upcoming 
task were presented above the item location for 3000 ms preceding each miniblock and remained on the screen throughout the miniblock.

Following the encoding phase, subjects completed the memory test which was organized into nine blocks of 48 trials each. Within a block, the first half of trials targeted one of the encoding conditions (e.g., artist) and the other half targeted the other condition (e.g., function). The order of conditions targeted was randomly chosen for each block, and subjects were explicitly instructed about which items were targeted prior to the start of each half. The instructional cue about which encoding condition was targeted was displayed above the item location for $6000 \mathrm{~ms}$ at the start of each half of trials and was continually displayed with the items. As an example, when the artist encoding condition was targeted, subjects were presented with an intermixed list of 12 items encoded with the artist task and 12 new items. Each item required a simple recognition judgment of whether the item was old or new, made by pressing the "m" and "," keys with the right index and middle fingers. Test items were displayed for 3000 $\mathrm{ms}$, preceded by a central red fixation cross for $1500 \mathrm{~ms}$, and followed by a central white fixation cross for a random interval between 2000 and 4000 ms. For the first test block, subjects were instructed to respond as quickly as possible and prior to the offset of the item (at $3000 \mathrm{~ms}$ ) but also with accuracy. For the eight remaining blocks, an auditory response cue consisting of a $500-\mathrm{Hz}$ tone was presented for $200 \mathrm{~ms}$ following a given delay after item onset. For these blocks, subjects were instructed to make their response as quickly as possible when hearing the tone, sacrificing accuracy when necessary. For the second through 
ninth test blocks, the tone delay was set relative to the subject's median response time for the first block. The respective delays across blocks were the median $+20 \%,+10 \%,+0 \%,-10 \%,-20 \%,-30 \%,-10 \%$, and $+10 \%$.

\subsubsection{EEG Acquisition and Processing}

EEG acquisition and processing was identical to the methods employed in Experiment 1.

\subsubsection{EEG Analysis}

Two pattern-classification methods were employed in Experiment 2. First, a classifier was trained on the pre-stimulus oscillatory activity from the encoding period and then tested on the recognition memory test blocks. This procedure was used to assess the extent to which encoding-related information was recapitulated in service of the separate recognition tests. A 'pattern' consisted of 2773 features (59 electrodes $\times 47$ frequency bands) and was submitted to a classification algorithm (L2-regularized logistic regression), separately for each subject. Accuracy was used as a metric for performance, where above-chance classification (binary; 50\%) was taken as an indicator of orienting to information from a certain encoding task. The second analysis method was largely similar to that of Experiment 1. Pattern-classification was used on data from the prestimulus period of test items to identify differences according to which encoding condition was targeted. Because the response-deadline procedure was adjusted for each test block, the first 4 trials (comprising 2 old and 2 new items) of each half of a block were excluded from the analysis. The cross-validation procedure 
was also adjusted by training a classifier on eight blocks and evaluating it on the remaining block. Statistical significance was again determined with a randompermutation procedure (Maris \& Oostenveld, 2007).

\subsection{RESULTS}

\subsubsection{Behavior}

The behavioral data for the targeted memory tests were analyzed separately for the first block, in which response accuracy and RT were balanced by the subject (i.e. no response deadline procedure was employed), and for the remaining eight blocks, each of which included a deadline. The behavioral measures for the first block are summarized in Table 2. These results were submitted to separate ANOVAs that included factors of targeted task (artist or function) and item type (old or new). Analysis of the response proportions revealed only a significant interaction between the two factors $\left(F_{1,28}=4.49, p=.043\right)$. Follow-up t-tests of item type for each targeted task gave rise to a significant effect for the artist task $\left(t_{28}=2.44, p=.021\right)$, in which correct responses were more frequent for old compared to new items, but there was no significant effect for the function task (t28 $=.43, \mathrm{p}=.67)$. For the corresponding RTs, which were computed relative to test-item onset in this first block, the ANOVA gave rise only to a significant main effect of item type $\left(F_{1,28}=15.88, p<.001\right)$, in which $R T s$ were shorter for old compared to new items. Because we used each subject's median RT for correct judgments from the first block (regardless of targeted task and old/new status) to determine the response deadlines in subsequent blocks, we provide the subject- 
wise medians and summary data in Figure 10A. As shown, the medians ranged from about 710 to $1754 \mathrm{~ms}$, with an across group average of $1096 \mathrm{~ms}$.

Table 2. Behavioral measures for Experiment 2

Mean (and SEM) proportions of correct responses and associated response times (RTs; in milliseconds) for the first block (with no response deadline) of the targeted memory test of Experiment 2

\begin{tabular}{|l|l|l|l|}
\hline Targeted task & Item type & Proportion correct & Correct RT \\
\hline Artist & Old & $.92(.02)$ & $1108(50)$ \\
\hline & New & $.86(.03)$ & $1246(58)$ \\
\hline Function & Old & $.89(.03)$ & $1134(44)$ \\
\hline & New & $.91(.02)$ & $1228(66)$ \\
\hline
\end{tabular}

The correct response proportions and associated RTs for the retrieval blocks employing response deadlines are respectively displayed in Figures 10B and 10C. These two measures were again analyzed with separate ANOVAs, as above, that each included an additional factor corresponding to test block (first through eighth). For the proportions, ANOVA gave rise to significant main effects of item type $\left(F_{1,28}=7.43, p=.011\right)$ and block $\left(F_{4.6,127.6}=7.86, p<.001\right)$, as well as an interaction between those two factors $\left(F_{5.2,145.8}=2.33, p=.044\right)$. As is apparent in Figure 11B, correct proportions were generally higher for old items relative to new items and decreased going from the initial to later blocks. Followup t-tests assessing the item type effect for each block gave some insight into the interaction, such that the effect was not reliable for the final two blocks (respectively, $t_{28}=.99$ and $.39, p=.33$ and .70 ) but was generally significant for 
the remaining blocks ( $\mathrm{t}_{28}$ range: 2.29 to 3.94 , p range: .03 to .0005 ; with the exception of fourth block, $\left.\mathrm{t}_{28}=1.84, \mathrm{p}=.076\right)$. Notably, correct response proportions did not substantially rebound during the last two blocks, in which the response deadline was relaxed. Although we anticipated that subjects might use the added time in these later blocks to focus on making more accurate responses, performance may have potentially succumbed to fatigue or interference from test items presented in the earlier blocks. Turning to the RTs for correct judgments, which were computed relative to the onset of the response deadline in contrast to the measures from the first (no-deadline) test block, subjects appeared to follow instructions by responding shortly after the deadline. As shown in Figure 10C, across all of the blocks, $82 \%$ of responses were made within $600 \mathrm{~ms}$ of the deadline. ANOVA of these RTs gave rise to a main effect of item type $\left(F_{1,28}=40.4, p<.001\right)$ and block $\left(F_{3.3,91.8}=97.57, p<.001\right)$, in addition to an item type $x$ block interaction $\left(F_{4.3,120.2}=4.73, p=.001\right)$. T-tests assessing the block effect for each item showed the interaction was driven primarily by older item responses being faster in second to seventh blocks (t28 range: 2.61:4.27, $p$ range: .01 to .000 ). The effect of item type did not hold for the last two blocks $(\mathrm{t} 28=.75$ and $1.09, \mathrm{p}=.46$ and .29$)$. 


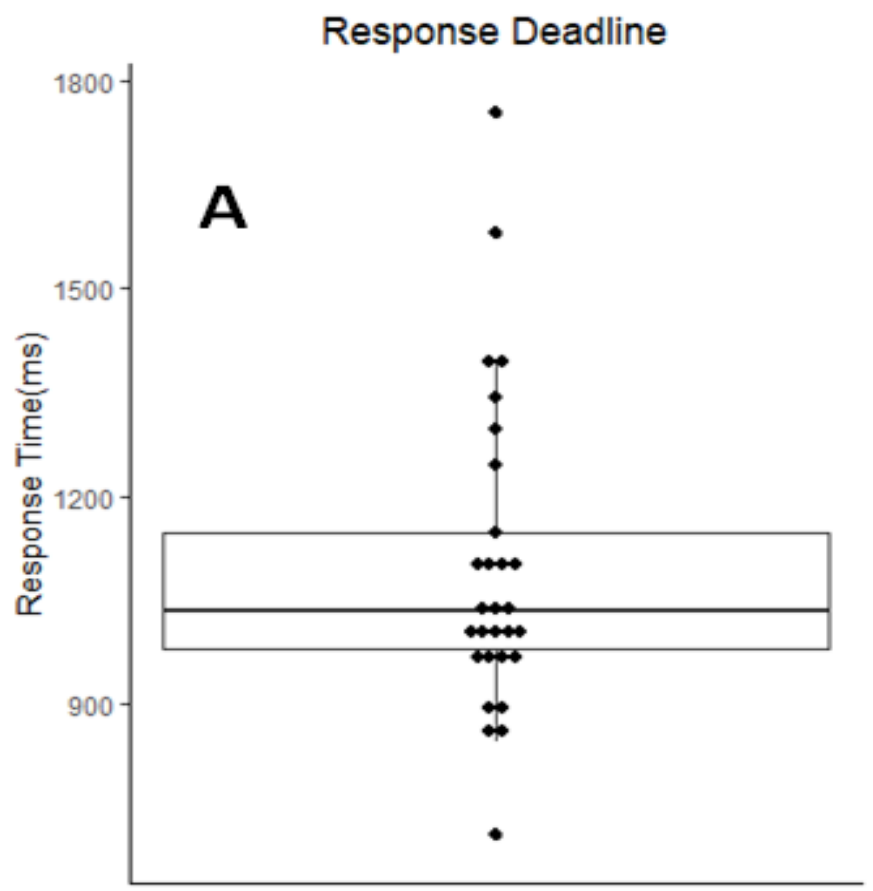

Deadline

B

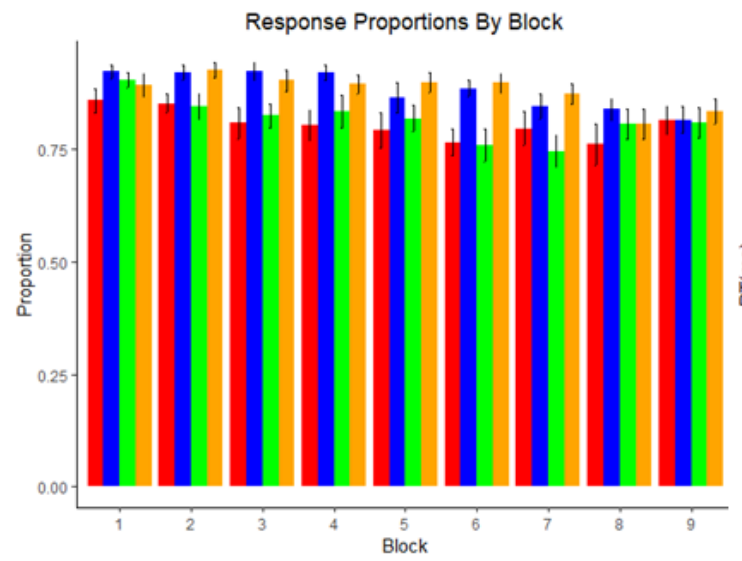

C

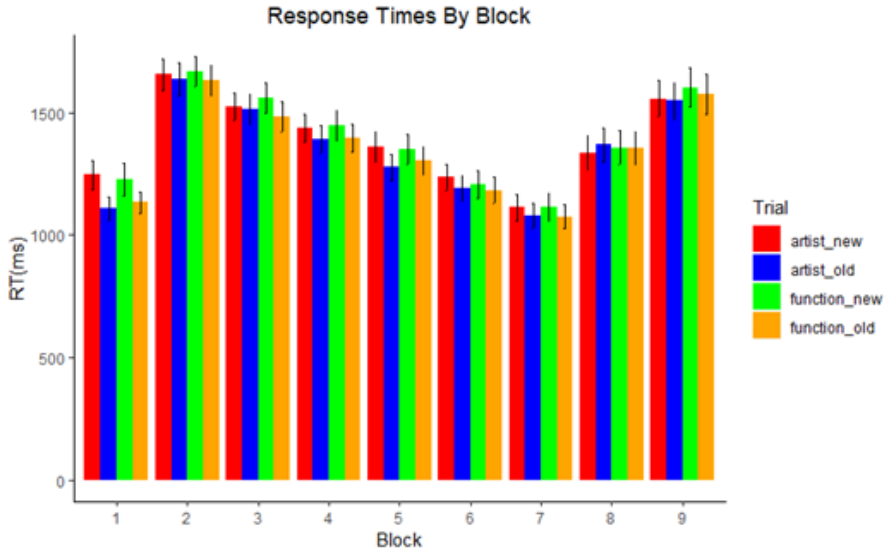

Figure 10. Behavioral measures for Experiment 2

A) Subject-wise median response times (RTs) from the first test block, which served as the basis for response deadlines in subsequent blocks. The box plot indicates the median, 25- and 75-\% intervals, and range of RTs.

B) Correct response proportions according to item type (old or new) and targeted task (artist or function) for each test block.

C) RTs corresponding to correct responses, according to item type (old or new) and targeted task (artist or function), for each test block. 


\subsubsection{EEG}

As described in the Introduction, one advance in experimental design introduced in the current experiment was the use of a single stimulus type (words) for the encoding phase. This feature not only allowed for employing a simple recognition task during the memory retrieval phase, but also allowed for using the EEG data from the encoding phase for classifier training (to distinguish the two encoding tasks). The main advantage of this design feature was that a large sample of uniform, task-related EEG data could be used for classifier training, while avoiding the use of data from the retrieval phase which was expected to change across test blocks. Below, the findings from this encoding-based classification analysis are reported first. To anticipate the findings, there was little evidence from this classification that subjects oriented differently to the two encoding conditions. To follow up on those initial results, we then report results from classification analyses that were based solely (i.e. both trained and tested) on the retrieval phase, analogous to the procedures of Experiment 1.

\section{Classification based on encoding activity}

The classifiers trained to distinguish the artist and function conditions based on encoding-phase data were first evaluated on retrieval data irrespective of the multiple test blocks. Figure 11A provides the results of this analysis when collapsing the EEG patterns over the entire pre-stimulus interval. As shown, classifier accuracy over all trials only reached .50 (SEM =.006), which did not differ significantly from the chance level of $.5\left(t_{27}=.25, p=.8\right)$. Segregating the 
trials according to item type (old and new) revealed comparable levels of accuracy, as shown in Figure 11B. Both of these measures were again statistically indistinguishable from chance (respectively, $\mathrm{M}=.51$ and .49 , SEM = .01 and $.01, \mathrm{t}_{27}=.85$ and $1.14, \mathrm{p}=.40$ and .26$)$.
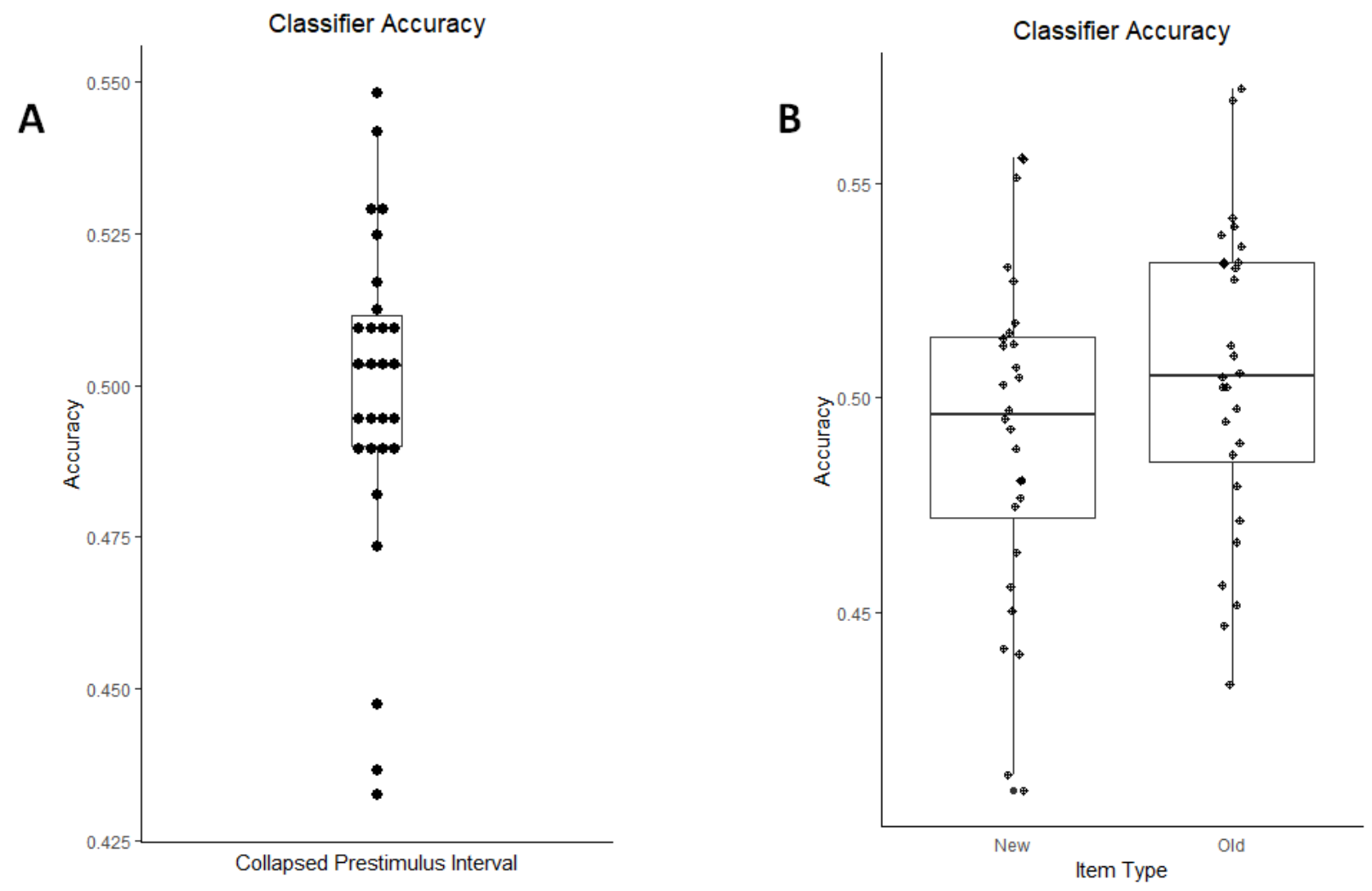

Figure 11. Pre-stimulus (collapsed) encoding-based classifier accuracy for Experiment 2

A) Classifier accuracy collapsed over the entire 1500-ms pre-stimulus interval and over all item types (old and new) in Experiment 2. The box plot indicates the median, 25- and 75-\% intervals, and range of accuracy values. Individual points correspond to the accuracy values for each subject.

B) Collapsed classifier accuracy determined in the same way as in Panel A, but segregated according to item type (old and new).

The trained classifier was next tested on data from each 100-ms bin during the pre-stimulus period to assess the time course of classification. The results of this 
analysis are provided in Figures $12 \mathrm{~A}$ and 12B, respectively, for all trials and according to old/new status. These analyses again demonstrated, as was evident in Experiment 1, that the levels of classifier accuracy were relatively flat. Contrary to those previous findings, however, the time courses for the current experiment remained near chance. Across the overall and item-type measures, accuracy for only the second and fourth time bins (i.e. 1400-1300 and 1200-1100 ms prior to item onset) even approached significance (respectively, $\mathrm{t}_{27}=1.8, \mathrm{p}=.075 ; \mathrm{t}_{27}=$ $1.2, p=.23)$, whereas the results for the remaining time bins were more clearly at chance (minimum $p=.14$ ). ANOVAs of classifier accuracy also failed to reveal a significant effect of time bin, either when all trials were collapsed $\left(F_{8.9,239.1}=.7, p\right.$ $=.71)$ or when item type (old or new) was included as a factor $\left(F_{7.2,194}=1.1, p=\right.$ .4). These findings thus fail to demonstrate any evidence of the artist/function distinction during the pre-stimulus test interval.

A

Classifier Time course

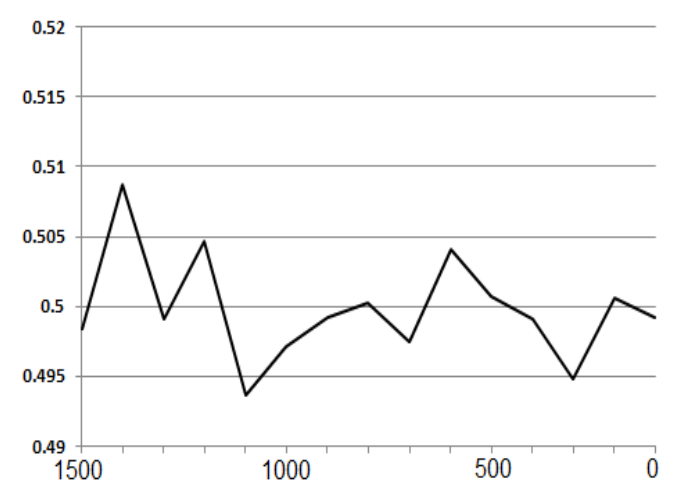

B Classifier Time course - By Item Type

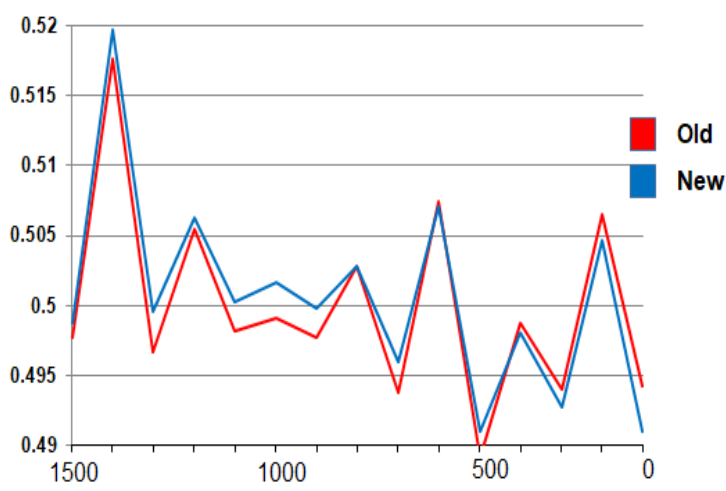

Figure 12. Pre-stimulus (uncollapsed) encoding-based classifier accuracy for Experiment 2

A) Group mean classifier accuracy by 100-ms time bins across the pre-stimulus interval, collapsed over all item types (old and new) in Experiment 2.

B) Group mean classifier accuracy determined in the same way as in Panel A, but segregated according to item type (old and new). 
Keeping with the analysis approach of Experiment 1, we next extracted the weights for each feature from the classifier trained on encoding data.

Segregating these data into the typical frequency bands of interest (theta, alpha, beta, and gamma) and contrasting them with a value of zero resulted in the topographic maps of $t$-values that are provided in Figure 13. As was stated before, the maps are displayed primarily for descriptive purposes. As shown, there appeared to be some consistency across the theta, alpha, and beta bands of positive weights for the artist task condition (negative for function task), and these important features were maximal near the midline. In the high-frequency, gamma band, positive weights were again evident for the artist task, but were focused over right-lateralized fronto-central sites. 


\section{Classifier Weights}

Theta $(4-8 \mathrm{~Hz})$
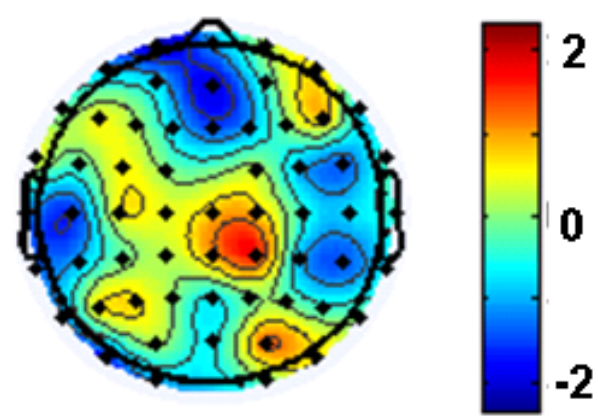

Beta $(13-30 \mathrm{~Hz})$

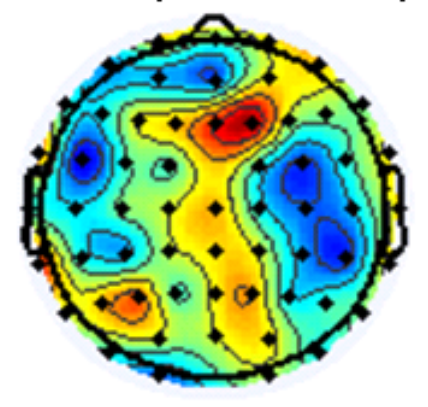

Alpha $(9-12 \mathrm{~Hz})$

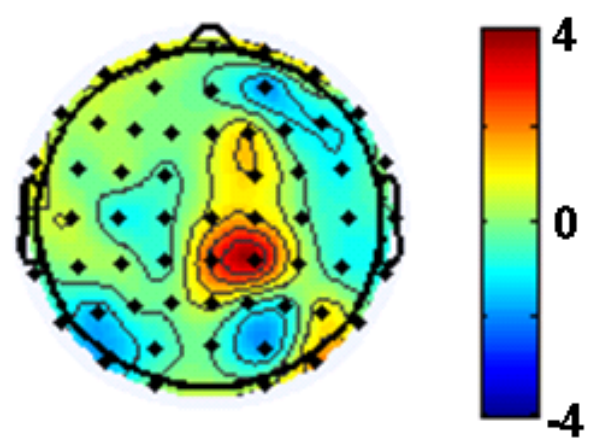

Gamma (31-50 Hz)

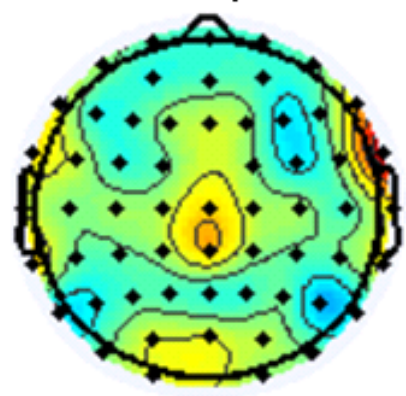

Figure 13. Topographic maps of encoding-based feature importance for

\section{Experiment 1}

Topographic maps of trained weights assigned to each feature, collapsed into traditional EEG frequency bands, and transformed into t-values (against 0 ). Warm colors represent positive importance values for targeting pictures (and negative for words), whereas cool colors represent positive importance values for targeting words (and negative for pictures).

Whereas the measures of classifier accuracy collapsed over all test blocks, as reported above, gave no indication that subjects were differentially orienting toward the artist and function encoding conditions, it remained possible that orienting was weak in some blocks and thereby diminished the overall accuracy 
measures. To test this possibility, the results of the above classification analyses were extracted separately for each block. The accuracy findings from the data collapsed over the entire pre-stimulus period are provided in Figure 14. As shown, accuracy for each block remained near chance (range $=.47$ to .53 ), with the maximal value occurring for the second block. This was the first block that employed the response deadline, but testing its accuracy against chance still failed to reveal a significant effect $\left(t_{27}=1.48, p=.15\right)$. An ANOVA of these results, employing a single factor for test block (first through ninth), again revealed no effect of block $\left(F_{6.3,169.4}=1.91, p=.31\right)$.

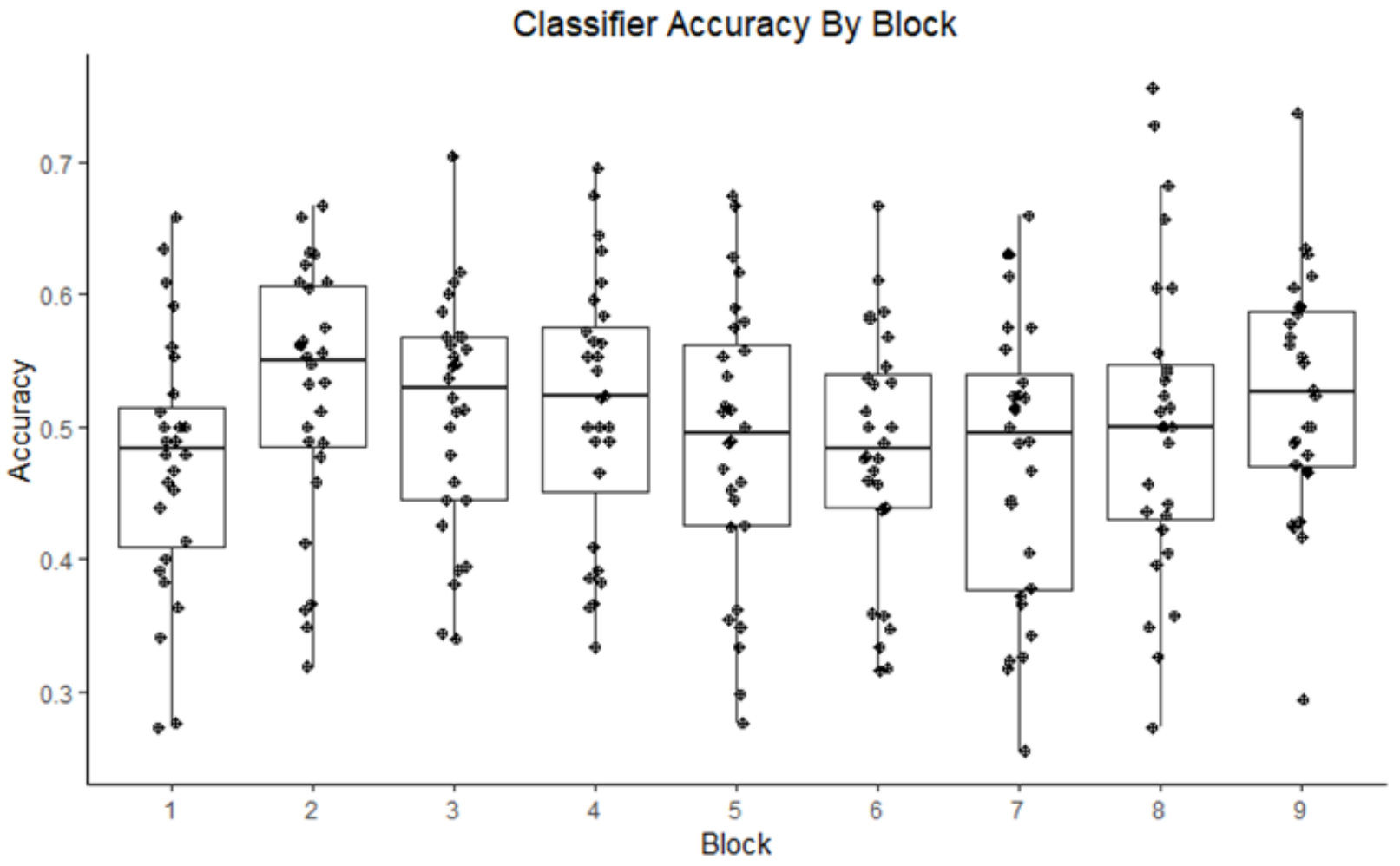

Figure 14. Pre-stimulus (collapsed) encoding-based classifier accuracy by test blocks in Experiment 2

Classifier accuracy collapsed over the entire 1500-ms pre-stimulus interval and over all item types (old and new) in Experiment 2, segregated according to test-block. The box plot indicates the median, 25- and 75-\% intervals, and range of accuracy values. Individual points correspond to the accuracy values for each subject. 
To probe further any potential changes in classifier accuracy across test blocks, we selected subsets of the data to identify possible trends. One trend was the change in accuracy from the second to seventh block, which would constitute evidence for our main hypothesis that increased demands to orient effectively were encouraged by shortening the response deadline. The accuracy values across these blocks were extracted for each subject, and a regression line was fit through each subject's values to determine a value for the slope. These subjectspecific slopes are displayed in Figure 15 and ranged from -.051 to .056 with mean of $-.01(\mathrm{SD}=.02)$. Despite being close to zero, testing the slopes means against that value revealed a significant difference $\left(t_{27}=2.58, p=.016\right)$, providing some evidence that accuracy changed with the main responsedeadline manipulation. Second, an analogous analysis was performed with the last two test blocks, where the response deadline was gradually relaxed, thereby making orienting less necessary. The subject-wise slopes for these data, shown in Figure 15 , had a mean of $.02(S D=.16$; range $=-.25$ to .34$)$, which was indistinguishable from zero $\left(\mathrm{t}_{27}=.77, \mathrm{p}=.45\right)$. 


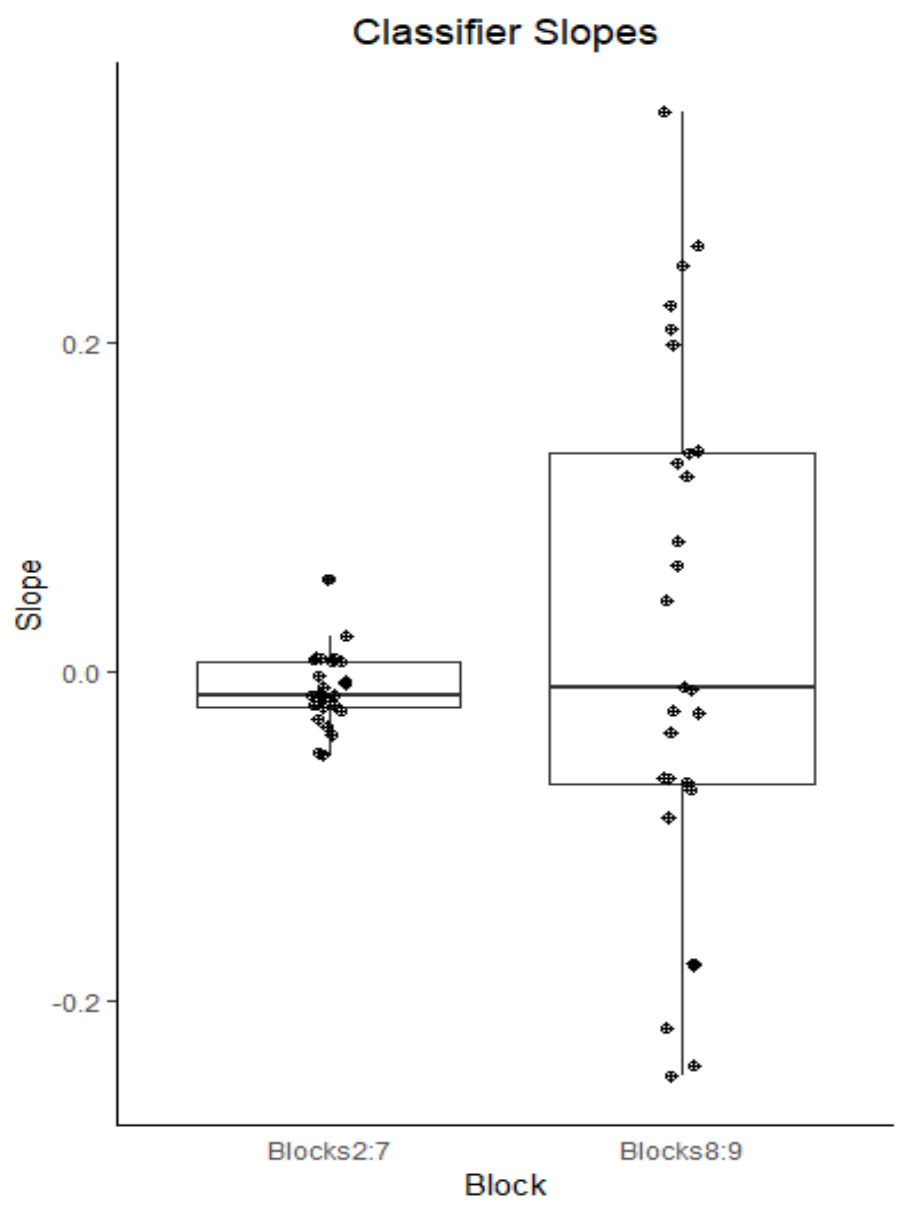

Figure 15. Slopes of encoding-based pre-stimulus classifier accuracy across test blocks of Experiment 2

Slopes determined by subject-wise regression analyses of classifier accuracy over subsets of test blocks. The box plot indicates the median, 25- and 75-\% intervals, and range of slopes. Individual points correspond to values for each subject.

To provide a complete picture of the results, we finally examined the time courses of classifier accuracy according to each of the test blocks. Given the findings of Experiment 1 and those of the current experiment when collapsing over blocks, in which accuracy was relatively flat over time, we expected a similar pattern of results here for each block. This expectation appeared to be borne out, as is displayed in Figure 16. Across blocks and pre-stimulus time, accuracy 
remained near chance and within a range from about .46 to .54 . Nonetheless, to test whether the time courses carried any information relevant to the artistfunction classification, we submitted these measures to an omnibus ANOVA that employed factors of test block (first through ninth) and time bin (fifteen 100-ms intervals). The ANOVA revealed no significant effects.

\section{Encoding Trained Classifier- By Block}
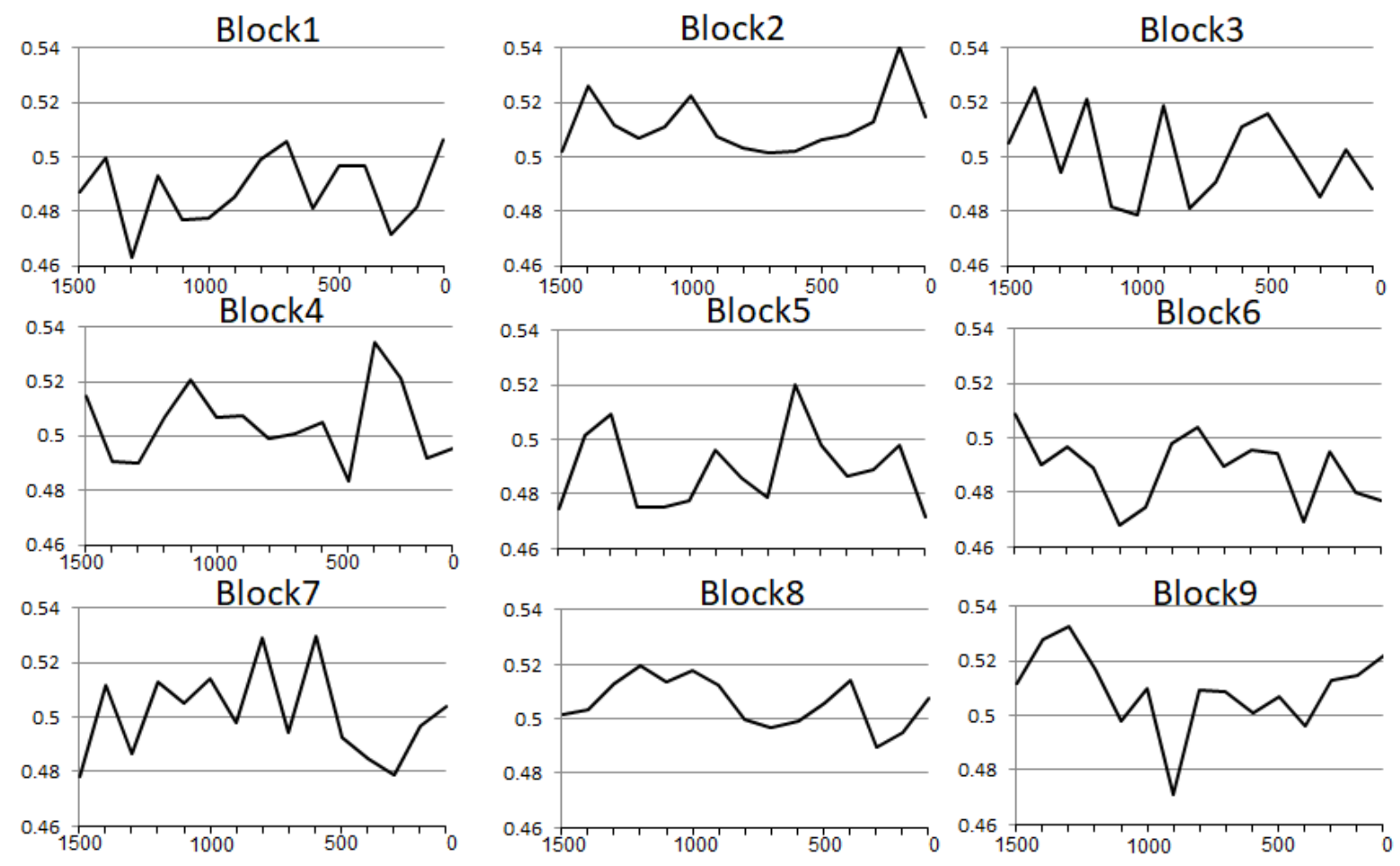

Figure 16. Pre-stimulus (uncollapsed) encoding-based classifier accuracy by test block in Experiment 2

Group mean classifier accuracy by 100-ms time bins across the pre-stimulus interval, collapsed over item type (old and new) and segregated according to test block. Chance $=.5$.

To ensure that the null findings reported above were not merely due to an absence of distinct patterns at encoding, the classification analysis was repeated by focusing only on data from the encoding phase. This analysis employed a 
cross-validation procedure in which the encoding data was divided into three blocks (72 trials per block). The data from two blocks were used for training, while that from the third block was left out for classifier evaluation; this process was then repeated to allow each block to be evaluated. While classifier accuracy during the encoding phase was somewhat unremarkable at a level of .53 , it was significantly above chance $\left(\mathrm{t}_{27}=3.38, \mathrm{p}=.002\right)$, indicating that task-specific neural patterns were elicited at encoding. Moreover, a correlation analysis of the subject-wise measures of classifier accuracy at encoding and retrieval revealed a negative and non-significant relationship $(r=-.23, p=.25)$. The absence of a positive correlation between these values thus suggests that classifier performance at retrieval was not simply a factor of some poorly-performing subjects.

\section{Classification based on retrieval activity}

Despite the largely-null findings of the above analyses, it is possible for a number of reasons that training the classifier on data from the encoding phase is not ideal. For example, it could be argued that the cognitive demands change in a substantial manner going from encoding to retrieval. These changes in turn could occlude some of the differences between the artist and function task conditions. Additionally, the fact that the two phases are wholly separated in time could cause any differences in noise (e.g., due to physiological or equipment factors) between the phases to be highlighted instead of the task conditions of interest. To accommodate these and other possibilities, we repeated the classifications by using only the data from the retrieval phase, analogous to what was done in 
Experiment 1. The classifiers for this analysis were trained on eight out of the nine test blocks and tested on the ninth block, while rotating the block that was tested (i.e. leave-one-block-out cross-validation). As noted earlier, including blocks in the training set where orienting may have been weak is likely not ideal, but the detrimental effects of doing so would hopefully be diminished by the inclusion of several "more ideal" blocks. The results of this classification when collapsing across the pre-stimulus period are provided in Figure 17A. As shown, accuracy remained near chance at a level of $.51\left(S D=.078 ; t_{27}=.68, p=.50\right)$. Similarly, the time course of accuracy, shown in Figure 17B, was again flat and at chance $\left(\right.$ maximum $\mathrm{t}_{27}=.57, \mathrm{p}=.58$ ).

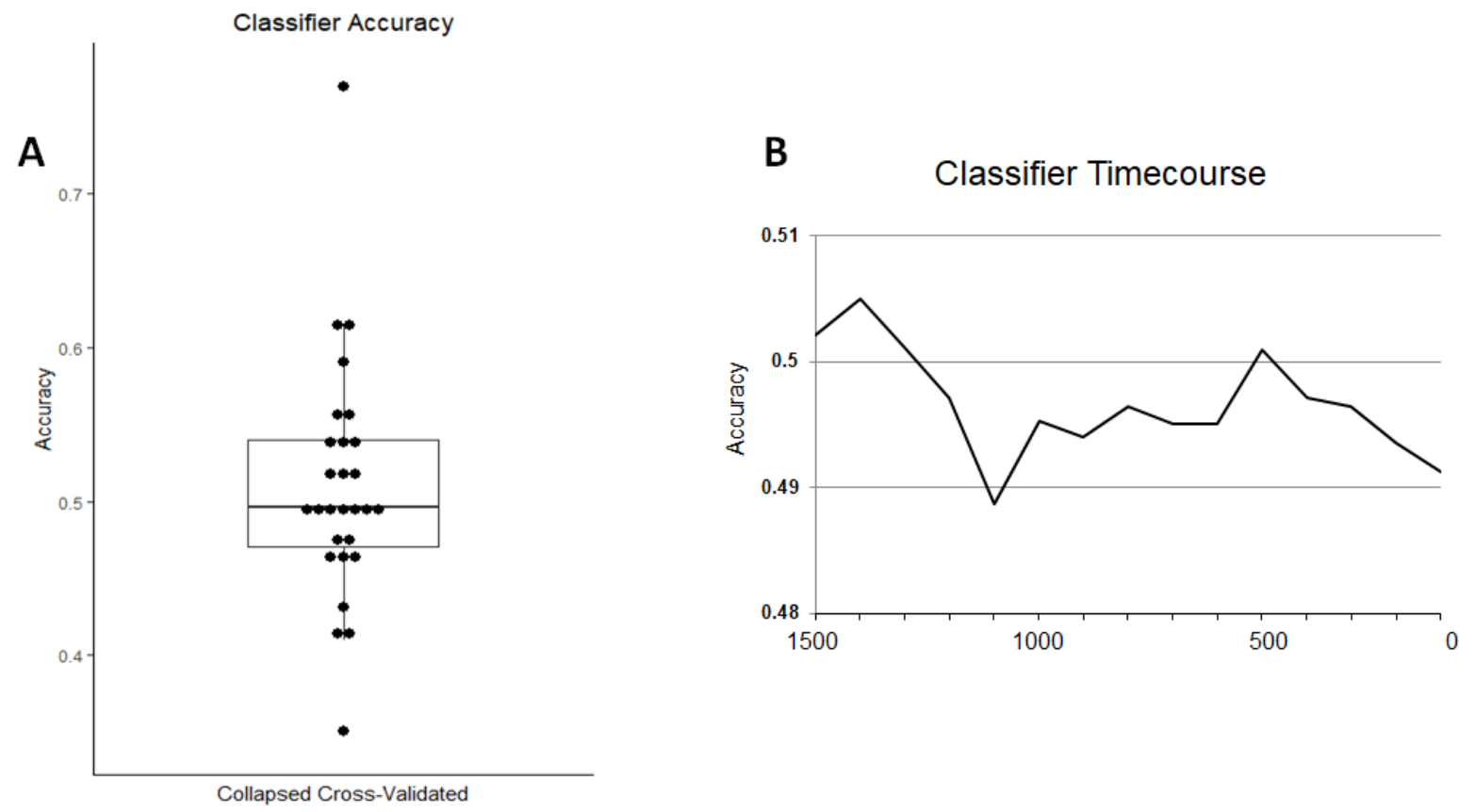

\section{Figure 17. Pre-stimulus retrieval-based classifier accuracy for Experiment 2}

A) Classifier accuracy collapsed over the entire 1500-ms pre-stimulus interval for correctlyrejected new items in Experiment 2. Data are also collapsed over all test blocks. The box plot indicates the median, 25- and 75-\% intervals, and range of accuracy values. Individual points correspond to the accuracy values for each subject.

B) Group mean classifier accuracy by 100-ms time bins across the pre-stimulus interval for correctly-rejected new items in Experiment 2. Data are also collapsed over all test blocks. 
Following the analyses of the encoding-based classification, we next tested the cross-validated retrieval-based classifier for any differences in accuracy across test blocks. Again, classification performance generally remained near chance levels, as is apparent in Figure 18A. Accuracy reached a maximum value of .55 $(\mathrm{SEM}=.02)$ in the fourth test block, but this result only marginally different from chance $\left(\mathrm{t}_{27}=1.83, \mathrm{p}=.08\right)$. An ANOVA of accuracy with test block (first through ninth) as a factor also failed to indicate any significant differences by block $\left(F_{6,161.9}=.92, p=.48\right)$. We also repeated the analyses of subject-wise slopes that were tested over subsets of test blocks as was done for the encoding-based classification. The results of these analyses are provided in Figures 18B and 18C. For second through seventh blocks, the mean slope was -.004 (SEM $=.01$, range $=-.08$ to .07 ; see Figure $18 \mathrm{~B}$ ). For the seventh through ninth blocks, the slope was $.02(S D=.14$, range $=-.25$ to .28 ; see Figure $18 C)$. Neither of these analyses indicated significant differences from zero (i.e. no block-related changes; respectively, $\mathrm{t}_{27}=.68$ and $.75, \mathrm{p}=.50$ and .46 ). 

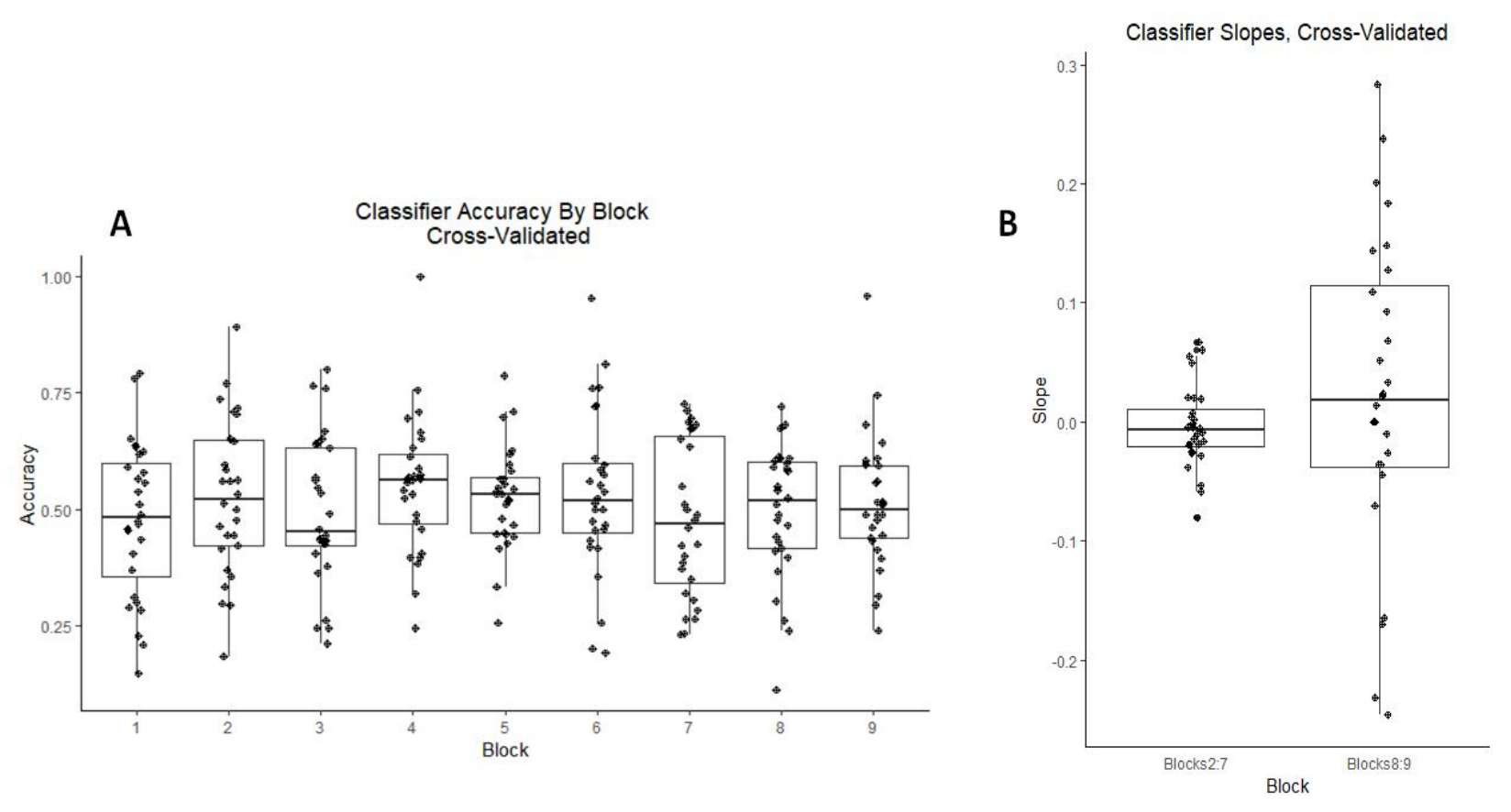

Figure 18. Pre-stimulus (collapsed) retrieval-based classifier results by test blocks in Experiment 2

A) Classifier accuracy collapsed over the entire 1500-ms pre-stimulus interval and over all item types (old and new) in Experiment 2, segregated according to test-block. The box plot indicates the median, $25-$ and $75-\%$ intervals, and range of accuracy values. Individual points correspond to the accuracy values for each subject.

B) Slopes determined by subject-wise regression analyses of classifier accuracy over subsets of test blocks. The box plot indicates the median, $25-$ and $75-\%$ intervals, and range of slopes. Individual points correspond to values for each subject.

Finally, the data from the pre-stimulus interval for each test block was divided into 100 -ms bins to further test the retrieval-trained classifier. The results of this classification are shown in Figure 19. As shown, the accuracy measures were again relatively flat across time and and across test block. An ANOVA confirmed this pattern by indicating no significant effects of either factor (respectively, $\left.F_{6.5,174.2}=.94, p=.47 ; F_{5.7,155.1}=1.43, p=.21\right)$. 
Cross-Validated Classifier - By Block
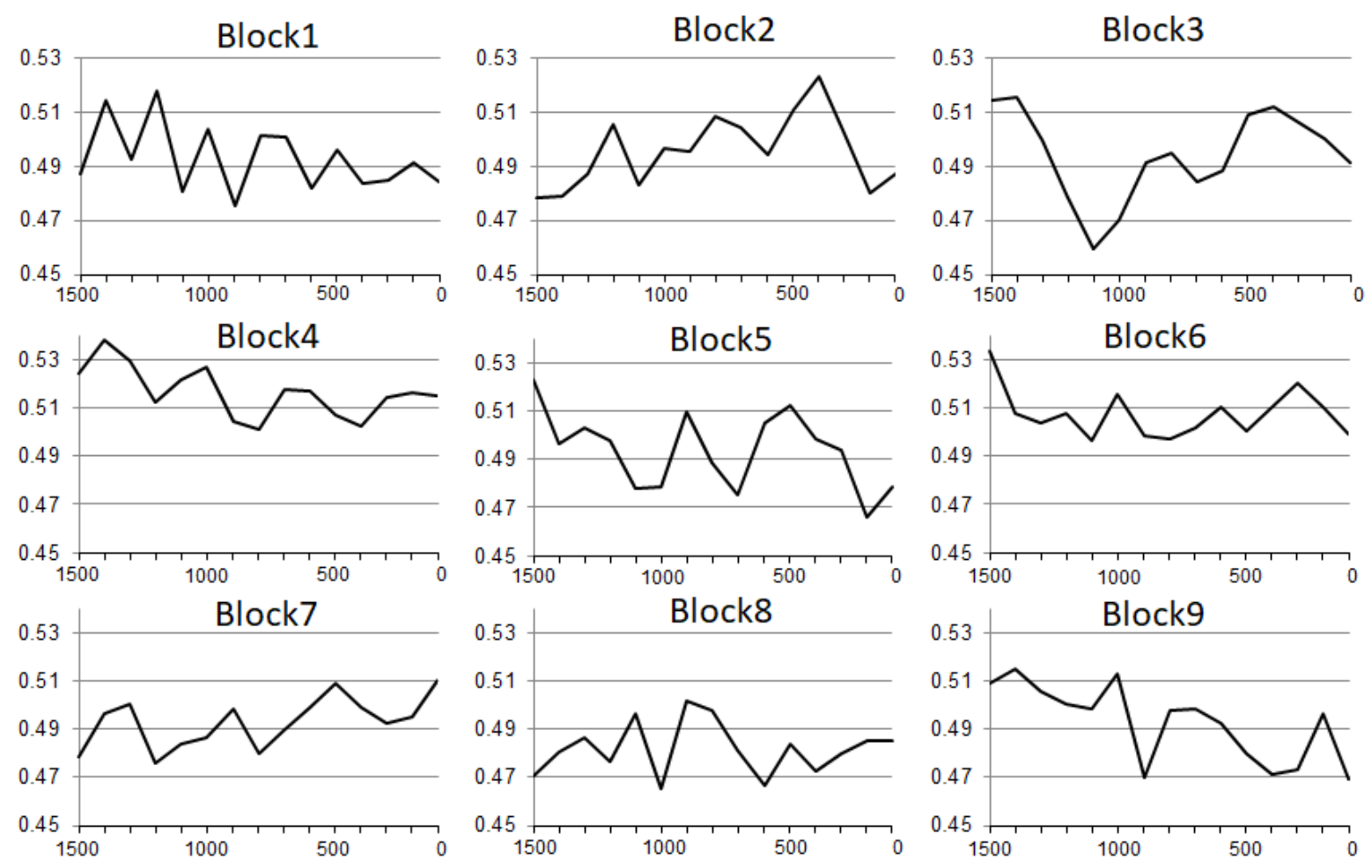

Figure 19. Pre-stimulus (uncollapsed) retrieval-based classifier accuracy by test block in Experiment 2

Group mean classifier accuracy by $100-\mathrm{ms}$ time bins across the pre-stimulus interval, collapsed over item type (old and new) and segregated according to test block. Chance $=.5$.

\subsection{DISCUSSION}

Experiment 2 sought to modulate the response demands of a memory retrieval task, with the goal of testing whether subjects likewise adapted their use of retrieval orienting strategies to maintain performance. Subjects encoded a list of words under two task instructions, and then completed a series of recognition memory tests that separately targeted the items from each instruction condition. Median RTs from the initial recognition memory tests were used to establish a 
level of baseline performance, from which a response deadline procedure for the remaining test blocks were based. As stated earlier, our main hypothesis was that orienting effects, as identified by the performance of a pattern classifier trained on encoding-phase EEG data, would increase in magnitude with the increased task demands of the shortened response deadline. Additionally, we aimed to test whether there were lasting effects of such demands after the deadline was relaxed or if orienting decreased in a corresponding manner. However, testing these hypotheses was precluded by the fact that classification accuracy was statistically at chance throughout the pre-stimulus period of recognition test trials. There was thus no evidence whatsoever that subjects differentially oriented to the encoding-task information during retrieval. Related to our hypotheses, classifier accuracy also remained largely unchanged despite the increase and subsequent relaxation of demands placed by the response deadline. As the RTs across blocks appeared to follow the deadline manipulation, we can conclude that subjects did follow the instructions put forth for the response deadline, despite the neural data exhibiting no related effects.

In order to attempt to rule out possible explanations of the aforementioned null findings, we repeated the MVPA procedure by cross-validating classifiers on EEG data solely from the retrieval phase. This procedure was analogous to that employed for Experiment 1, in which we found reliable evidence of differential orienting, and was expected to minimize any differences due to switching between separate experimental phases for classifier training and testing. In the end, however, the null findings remained. We return to other potential 
explanations of these findings, in the context of evidence for orienting obtained from Experiment 1, in the next section. 


\section{GENERAL DISCUSSION}

As described in the Introduction, numerous studies have employed ERPs to investigate 'retrieval orientation', a phenomenon which refers to the cognitive and neural processes engaged during episodic memory retrieval in service of increasing the likelihood of access to a particular type of mnemonic information (e.g., Ranganath \& Paller, 1999; Robb \& Rugg, 2002; Herron \& Rugg, 2003; Herron \& Wilding, 2004, 2006; Werkle-Bergner et al., 2005; Johnson \& Rugg, 2006a, 2006b; Bridger \& Mecklinger, 2012; Rosburg et al., 2014). Despite the consistent evidence that subjects differentially orient retrieval processing across a variety of manipulations (e.g., stimulus material and encoding-task operations), there are some important shortcomings to the approach of relying on the analysis of ERP amplitudes for such evidence. As we have laid out here, one of these shortcomings is that the analyses focus entirely on neural activity from the poststimulus period (i.e. after the test-item onsets), thereby running the risk of conflating any effects of differential orienting with those related to retrieval success of different memory traces or subsequent post-retrieval processing (e.g.,

Wilding \& Rugg, 1996; Wilding, 1999; Friedman \& Johnson, 2000; for review, see Rugg \& Wilding, 2000). To address this problem, studies have largely taken the approach of restricting analyses to the ERPs elicited by correctly-rejected new test items, which should be associated with minimal neural activity related to successful retrieval. Doing so, however, reduces the amount of EEG data that can contribute to the findings, thus limiting the statistical power of the analysis. Furthermore, another shortcoming is that analyses of ERP amplitude require that 
the waveforms be baseline-corrected in order to reduce the effects of lowfrequency drift as well as the carry-over of processing on prior trials. Because baseline-correction is typically based on the pre-stimulus interval, though, any differences in orienting during that period, which would presumably reflect preparatory processes, would be occluded. In the current project, a pair of experiments were conducted in attempt to circumvent each of these issues and further develop an analysis approach that is beneficial for investigating retrieval orientation with EEG measures.

Both of the experiments described here utilized oscillatory activity (i.e. power at specific frequency bands across time) in combination with multivariate pattern analysis (MVPA) to quantify the extent to which subjects were orienting to specific types of encoded information. MVPA has been widely used with data from functional magnetic resonance imaging (fMRI) in recent years (see Haynes \& Rees, 2005; Norman et al., 2006; for reviews, see Rissman \& Wagner, 2012; Tong \& Pratt, 2012), and its use with EEG (and likewise, magnetoencephalography) data is rapidly increasing, particularly in the context of episodic memory (e.g., Jafarpour et al., 2014; Johnson et al., 2015; Morton \& Polyn, 2017). In the context of the present experiments, MVPA was used to determine whether information related to a specific encoding condition being targeted at the time of retrieval was present in the patterns of oscillatory activity prior to test-item onset (i.e. during the pre-stimulus period). A general prediction we held was that when information specific to a particular condition - picture versus word materials during Experiment 1, and artist versus function tasks in 
Experiment 2 - was being targeted, a pattern classifier trained to distinguish such conditions would be accurate at making the same distinction with an independent (non-trained) set of EEG data.

In Experiment 1, an exclusion procedure was used during the memory test, in which subjects had to respond positively to items from one category (e.g., encoded pictures) while responding negatively to new items as well as those from the opposing, non-targeted category (encoded words in this example). Analysis of the behavioral data revealed that accuracy and RTs were similar across the two targeting conditions, allowing us to rule out any potential confounding effects on the neural data due to differences in difficulty or effort. MVPA of the patterns of oscillatory activity during the pre-stimulus interval indicated that the information being targeted during retrieval could be accurately decoded, providing novel evidence that subjects strategically adapted retrieval processing in a preparatory manner to potentially influence the processing of the upcoming retrieval cues and thereby the likelihood of retrieval success. Classifier accuracy also exhibited a sustained pattern throughout the 1500-ms pre-stimulus interval, suggesting that subjects adopted orienting-related processing in a preparatory manner and maintained such processing akin to engaging in a prolonged cognitive state. Further evidence for sustained processing was provided by similar classification results obtained for the EEG data from the poststimulus interval.

One other notable finding from Experiment 1 came from the analyses that were conducted to identify features important to classifying pictures versus words. In 
one form of these analyses, separate classifications were carried out for the electrode-wise patterns of activity at each time bin (100-ms intervals) and frequency band (see Figures 5 and 9). Despite the activity at lower frequencies contributing to above-chance classifier performance, as was evident during the pre-stimulus interval but to a lesser extent during the post-stimulus interval, those frequencies appeared to be only marginally driving classifier performance. Instead, maximal classifier accuracy was evident when based on the oscillatory activity at higher frequencies, particularly in the high beta and low gamma ranges. Given that the results of ERP amplitude analyses are typically dominated by low-frequency activity, likely at frequencies even lower than the $4-\mathrm{Hz}$ cutoff employed here, these contrasting effects from the classification analyses indicate that oscillatory activity provides a qualitatively distinct form of evidence for retrieval orientation. The two types of effects thus may rely on separate underlying neural mechanisms, suggesting that the advance in using MVPA of EEG oscillations may provide key insights into the further study of strategic processes that support memory retrieval.

In Experiment 2, the behavioral procedure was modified to incentivize the adoption of differential orienting to meet the demands of the memory retrieval task. Subjects encoded words under different task instructions and then completed a series of recognition test blocks that separately targeted items from each task condition. An overall measure of median response time (RT) during the first test block, in which subjects were free to balance the accuracy of their responses against speed, was used to establish a baseline of performance. This 
baseline then served as a starting point on which the response demands employed in the subsequent blocks were based. The response-deadline procedure introduced here comprised an auditory tone that was presented a given amount of time after the onset of the test item and indicated that subjects should make their responses as quickly as possible, sacrificing accuracy when necessary. In particular, the deadline was parametrically shortened across a series of six test blocks, with each consecutive block taking off an additional $10 \%$ of the median RT that was initially established in the first block. For the final two test blocks, the response deadline was relaxed in increments of $20 \%$ of the median RT value. Such within-session and subject-specific adaptive techniques have recently been used successfully to assess attentional control and mind wandering in cognitive and neuroimaging studies (e.g., deBettencourt et al., 2005; deBettencourt, Norman, \& Turk-Browne, 2017), but the application of these techniques here to episodic memory retrieval is novel.

Our main predictions for the results of Experiment 2 were that shortening the response deadline would serve as motivation for subjects to more effectively engage in differential orienting processing for the two classes of encoding items, thus increasing (or at least, maintaining) the likelihood of successful retrieval in the shortened time frames. To this end, the pre-stimulus oscillatory patterns were submitted to classifiers that were trained to distinguish items presented in the two task conditions at encoding. However, in stark contrast to the significant findings of Experiment 1, classifier accuracy remained at chance levels across a variety of different analysis approaches that were conducted in attempt to identify any 
evidence of distinct retrieval orienting. To account for potential issues with using a classifier trained on separate tasks (i.e. encoding versus retrieval) and separated by a substantial period of time, an additional set of classifiers were trained and tested in a cross-validation scheme on data taken solely from the retrieval test blocks (as was the case in Experiment 1). Nevertheless, performance remained statistically indistinguishable from the chance level. Thus, contrary to our expectations based on the findings of Experiment 1, there appeared to be no evidence that subjects differentially oriented to the two encoding conditions. Furthermore, the lack of modulation in orienting effects across test blocks goes against our main prediction and suggests a possible limit in the ability to flexibly adapt strategic orienting to meet task demands.

The discrepant findings between Experiments 1 and 2 could potentially be attributed to a number of differences in the procedures and materials employed. First, the stimuli employed in Experiment 2 were solely words (encountered under different encoding task instructions), whereas Experiment 1 used both word and picture stimuli. Visually-presented words and pictures are known to elicit distinct ERPs (e.g., Kutas \& Federmeier, 2011; Villena-Gonzalez et al., 2016), and thus correspondingly distinct cognitive processing, which could have facilitated the subsequent engagement of differential orienting processes at the time of retrieval. Nonetheless, this difference is unlikely to account entirely for the discrepant results, as multiple encoding tasks employed for a single stimulus type have been shown to give rise to retrieval orientation differences in prior studies (e.g., Dzulkifli \& Wilding, 2004; Herron \& Wilding, 2006). Another possible 
explanation of the discrepancy is due to the general differences in demands of the retrieval tasks across experiments, irrespective of the demands we sought to modulate with the response-deadline procedure. Namely, the exclusion task employed in Experiment 1 likely encouraged the retrieval of highly-specified information from encoding, so as to recover the source information related to targets while rejecting both non-targets and new items. By contrast, accurately responding to targets from the two encoding classes in Experiment 2 could have potentially been achieved by a general recognition strategy in which all old items were retrieved in the same manner. Although previous studies have found evidence of differential orienting in the context of recognition tasks (e.g., Robb \& Rugg, 2002; Johnson \& Rugg, 2006b; Roberts et al., 2014), such effects have been shown to be significantly weaker than those elicited by exclusion task procedures (Johnson \& Rugg, 2006b). A final feature of Experiment 2 that may have led to its null results was the shortened length of test blocks (24 trials each) implemented for targeting each encoding class. Previous studies have used much longer blocks, with Experiment 1 of the current project having subjects orient to a single class for 75 trials at a time. If one assumes that orienting retrieval takes the form of a sustained cognitive state, as the results of Experiment 1 suggest, then it could be the case that the length of the test blocks employed in Experiment 2 was too short. Whereas it is possible that none of these differences between experiments account solely for the discrepant findings, their combined existence could have contributed to the null results observed in Experiment 2. 
Despite the two experiments reported here constituting only partial support for oscillation-based retrieval orientation effects, the focus on pre-stimulus activity allows us to speculate about how new avenues for intervention may be developed. For example, the vast majority of memory-related interventions and rehabilitation techniques employed in older adult subjects have thus far focused on increasing the use of elaborative processing at encoding (e.g., Kirchhoff et al., 2012; Engvig et al., 2012). These studies follow findings that such processing strategies are impaired, or at least not regularly relied upon, in elderly individuals (e.g., Logan et al., 2002; Duverne et al., 2009; Grady, 2012). While informationspecific processing in support of episodic retrieval is difficult to assess in behavioral paradigms alone, future studies employing MVPA in combination with training or neurofeedback techniques may allow for more precise modulation of specificity-based (i.e. recollective) memory retrieval, thus mitigating age-related decline in episodic memory function. Clearly, based on the results of Experiment 2 alone, the methods designed to modulate strategic orienting need to be reconsidered, particularly in light of simple memory tasks (i.e. recognition) that older populations can successfully perform. Nevertheless, the findings of Experiment 1 provide a novel starting point for testing retrieval processes with pre-stimulus patterns of oscillatory activity, while both maximizing the amount of data that can be analyzed and allowing for individual-subject variation in the neural correlates of retrieval orientation.

As stated in the Introduction, one motivation for the current experiments was the fact that many prior attempts to train subjects with methods intended to boost 
memory performance focused on interventions at the encoding stage. To thoroughly explore the potential of effective training paradigms, a focus on processes acting at the time of retrieval is also necessary. Furthermore, before effective retrieval-based training becomes possible, the discrete stages of retrieval processing must be clearly demarcated. In Experiment 1 reported here, a pre-stimulus signature of selectively orienting retrieval processes toward different encoded materials was established. In the future, effects analogous to these may be leveraged to identify deficits of cognitive control in clinical or preclinical populations that may be susceptible to cognitive decline. Additionally, real-time measurement of orienting-related signals, such as those that can be obtained with EEG oscillatory activity, may become central in experimental paradigms involving neurofeedback, thereby assisting subjects in flexibly modulating cognitive control processes that support memory retrieval. Given that oscillatory activity in the higher frequency ranges (i.e. beta and gamma bands) was found to drive classifier performance in Experiment 1, classification of these signals could become possible at timescales that would allow for within-session changes that might not be observable using other neuroimaging methodologies, such as fMRI (e.g., deBettencourt et al., 2015). Paradigms such as that employed here may also aid in refining cortical patterns that become dedifferentiated in older adults by tuning function in those regions. Furthermore, the techniques enlisted in Experiment 1 allow for probing the relationship between material-specific representations and behavior. With that goal in mind, it is important to note the limitations of this technique that were revealed in 
Experiment 2. More generalized forms of memory, often spared in older adults, may not elicit the required signature of orienting that must be leveraged.

Broadly, the techniques utilized in the current study, and the use of patternclassification analyses of EEG oscillatory activity in particular, could be easily adapted to other experimental contexts to investigate memory function and dysfunction across a variety of populations. Such data are crucial in forming a more coherent picture of the relationship between brain oscillations and behavioral performance. For example, under the assumption that synchronous activity in the gamma frequency band is conducive to retrieval success, developing pharmacological therapies or stimulation paradigms that selectively boost such synchrony may have important effects in mitigating the memory deficits commonly evident with elderly adults. In combination with responsemodulation paradigms such as that employed in Experiment 2, patient-specific interventions in which the specific rhythms and regions that may be selectively responsible for impairment could be better targeted. For instance, GABA has been linked to inhibitory control and has been shown to be relatively decreased in older adults, particularly in dorsal anterior cingulate cortex (Marenco et al., 2018). Given that inhibitory control could underlie the overgeneralized memory patterns often seen in older adults, pharmacotherapies combined with neurofeedback may provide gains not manifest in isolation. In closing, multivariate pattern analyses of cognitive processes that behavioral paradigms leave ambiguous allows for the advancement of both theory and practice in resolving or rehabilitating functions that may decline due to age or other clinical deficits. 


\section{REFERENCES}

Addante, R. J., Watrous, A. J., Yonelinas, A. P., Ekstrom, A. D., \& Ranganath, C. (2011). pre-stimulus theta activity predicts correct source memory retrieval. Proc. Nat. Acad. Sci., 108(26), 10702-10707.

Bridger, E. K. \& Mecklinger, A. (2012). Electrophysiologically dissociating episodic preretrieval processing. J. Cogn. Neurosci, 24(6), 1476-1491.

Bridger, E. K., Sprondel, V., \& Mecklinger, A. (2015). Control over recollection varies with context-type: ERP evidence from the exclusion task. Cogn. Neurosci., 6(1), 31-38.

Chaumon, M., Bishop, D. V., \& Busch, N. A. (2015). A practical guide to the selection of independent components of the electroencephalogram for artifact correction. J. Neurosci. Methods, 250, 47-63.

deBettencourt, M. T., Cohen, J. D., Lee, R. F., Norman, K. A., \& Turk-Browne, N. B. (2015). Closed-loop training of attention with real-time brain imaging. Nature Neurosci., 18(3), 470-475.

deBettencourt, M. T., Norman, K. A., \& Turk-Browne, N. B. (2017). Forgetting from lapses of sustained attention. Psych. Bull. Rev., 2017.

Delorme, A. \& Makeig, S. (2004). EEGLAB: an open source toolbox for analysis of single-trial EEG dynamics including independent component analysis. J. Neurosci. Methods, 134, 9-21. 
Duverne, S., Motamedinia, S., \& Rugg, M. D. (2009). Effects of age on the neural correlates of retrieval cue processing are modulated by task demands. J. Cogn. Neurosci., 21(1), 1-17.

Duzel, E., Cabeza, R., Picton, T. W., Yonelinas, A. P., Scheich, H., Heinze, H., \& Tulving, E. (1999). Task-related and item-related brain processes of memory retrieval. Proc Nat. Acad. Sci, 96(4), 1794-1799.

Dzulkifli, M. A. \& Wilding, E. L. (2005). Electrophysiological indices of strategic episodic retrieval processing. Neuropsychologia, 43, 1152-1162.

Dzulkifli, M. A., Sharpe, H. L., Wilding, E. L. (2004). Separating item-related electrophysiological indices of retrieval effort and retrieval orientation. Brain Cogn., 55, 433-443.

Eich, J. E., Weingartner, H., Stillman, R. C., \& Gillin, J. C. (1975). Statedependent accessibility of retrieval cues in the retention of a categorized list. J. of Verb. Learn. \& Verb. Behav., 14, 408-417.

Engvig, A., Fjell, A. M., Westlye, L. T., Moberget, T., Sundseth, Ø., Larsen, V. A., \& Walhovd, K. B. (2012). Memory training impacts short-term changes in aging white matter: a longitudinal diffusion tensor imaging study. Human Brain Mapping, 33(10), 2390-2406.

Fell, J., \& Axmacher, A. (2011). The role of phase synchronization in memory processes. Nat. Rev. Neurosci., 12, 105-118. 
Friedman, D., Johnson, J. R. (2000). Event-related potential (ERP) studies of memory encoding and retrieval: a selective review. Microsc. Res. Tech., 51, 628.

Fuentemilla, L., Palombo, D. J., \& Levine, B. (2018). Gamma phase-synchrony in autobiographical memory: Evidence from magnetoencephalography and severely deficient autobiographical memory. Neuropsychologia, 110, 7-13.

Gao, C., Rosburg, T., Hou, M., Li, B., Xiao, X., Guo, C. (2016). The role of retrieval mode and retrieval orientation in retrieval practice: insights from comparing recognition memory testing formats and restudying. Cogn Aff. Behav. Neurosci., 16, 977-990.

Gruber, M. J. Watrous, A. J., Ekstrom, A. D., Ranganath, C., \& Otten, L. J. (2013). Expected reward modulates encoding-related theta activity before an event. Neuroimage, 64, 68-74.

Grady, C. (2012). "The Cognitive Neuroscience of Ageing." Nature Reviews. Neuroscience 13 (7): 491-505.

Hanslmayr, S., Spitzer, B., \& Bauml, K.H. (2009). Brain oscillations dissociate between semantic and nonsemantic encoding of episodic memories. Cerebral Cortex, 19(7), 1631-1640.

HansImayr, S., Staudigl, T. (2014). How brain oscillations form memories - A processing based perspective on oscillatory subsequent memory effects.

Neuroimage, 85, 648-655 
Hanslmayr, S., Staresina, B. P., \& Bowman, H. (2016). Oscillations and episodic memory: addressing the synchronization/desynchronization conundrum. Trends in Neurosciences, 39(1), 16-25.

Haynes, J. D., \& Rees, G. (2006). Decoding mental states from brain activity in humans. Nat. Rev. Neurosci., 17, 523-534.

Herron, J. E. \& Rugg, M. D. (2003). Retrieval orientation and the control of recollection. J. of Cogn. Neurosci., 15, 843-854.

Herron, J. E. \& Rugg, M. D. (2003). Strategic influences on the recollection in the exclusion task: electrophysiological evidence. Psychon. Bull. Rev., 10, 703-710.

Herron, J. E. \& Wilding, E. L. (2004). An electrophysiological dissociation of retrieval mode and retrieval orientation. Neuroimage, 22, 1554-1562.

Herron, J. E. \& Wilding, E. L. (2006). Neural correlates of control processes engaged before and during recovery of information from episodic memory. Neuroimage, 30, 634-644.

Herron, J. E., Evans, L. H., Wilding, E. L. (2016). Electrophysiological evidence for flexible goal-directed cue processing during episodic retrieval. Neuroimage, 132, 24-31.

Hornberger, M., Rugg, M. D., \& Henson, R. N. (2006). ERP correlates of retrieval orientation: direct versus indirect memory tasks. Brain. Res., 1071(1),124-136. 
Jacoby, L. L. (1991). A process dissociation framework: separating automatic from intentional uses of memory. J. Mem. Lang., 30, 513-541.

Jacoby, L. L., Shimizu, Y., Daniels, K. A., \& Rhodes, M. G. (2005). Modes of cognitive control in recognition and source memory: depth of retrieval. Psychon. Bull. Rev., 12, 852-857.

Jafarpour, A., Fuentemilla, L., Horner, A., Penny, W., and Duzel, E. 2014. "Replay of Very Early Encoding Representations during Recollection." The Journal of Neuroscience34 (1): 242-48.

Johnson, J. D. \& McGhee, A. K. (2015). Electrophysiological evidence for strategically orienting retrieval toward the specific age of a memory. Brain and Cognition, 100, 41-48.

Johnson, J. D. \& Rugg, M. D. (2006a). Electrophysiological correlates of retrieval processing: effects of consistent versus inconsistent retrieval demands. J. of Cogn. Neurosci., 18(9), 1531-1544.

Johnson, J. D. \& Rugg, M. D. (2006b). Modulation of the electrophysiological correlates of retrieval cue processing by the specificity of task demands. Brain Research, 1071, 153-164.

Jung, T. P., Makeig, S., Westerfield, W., Townswend, J., Courchesne, E., \& Sejnowski, T. J. (2000). Removal of eye activity artifacts from visual eventrelated potentials in normal and clinical subjects. Clin. Neurophys., 111, 17451758. 
Keating, J., Affleck-Brodie, C., Wiegand, R., Morcom, A. M. (2017). Aging, working memory capacity and the proactive control of recollection: An eventrelated potential study. PLOS ONE, 12(7):e0180367.

Kirchhoff, B. A., B. A. Anderson, D. M. Barch, and L. L. Jacoby. 2012. "Cognitive and Neural Effects of Semantic Encoding Strategy Training in Older Adults." Cerebral Cortex 22 (4): 788-99.

Klimesch, W., Schimke, H., Schwaiger, J. (1994). Episodic and semantic memory: an analysis in the EEG theta and alpha band. Electroencephal. Clin. Neurophys., 91, 428-441.

Klimesch, W. (1999). EEG alpha and theta oscillations reflect cognitive and memory performance a review and anlysis. Brain Research Reviews, 29(23),169-195.

Kutas, M., and Federmeier, K. (2011). Thirty Years and Counting: Finding Meaning in the N400 Component of the Event-Related Brain Potential (ERP). Annual Review of Psychology 62: 621-47.

Lega, B., Burke, J., Jacobs, J., \& Kahana, M. J. (2016). Slow-theta-to-gamma phase-amplitude coupling in human hippocampus supports the formation of new episodic memories. Cerebral Cortex, 26(1), 268-278.

Lisman, J. E., \& Jenson, O. (2013). The theta-gamma neural code. Neuron, 77(6), 1002-1016. 
Logan, J. M., Sanders, A.L., Snyder, A.Z.,Morris, J.C., and Buckner,R.L. (2002). Under-Recruitment and Nonselective Recruitment: Dissociable Neural Mechanisms Associated with Aging. Neuron 33 (5): 827-40.

Long, N. M., Burke, J. F., \& Kahana, M. J. (2014). Subsequent memory effect in intracranial and scalp EEG. Neuroimage, 84, 488-494.

Marenco, S., Meyer, C., van der Veen, J. W., Zhang, Y., Kelly, R., Shen, J., ... Berman, K. F. (2018). Role of gamma-amino-butyric acid in the dorsal anterior cingulate in age-associated changes in cognition. Neuropsychopharmacology 43(11), 2285-2291.

Maris, E., \& Oostenveld, R. (2007). Nonparametric statistical testing of EEG and MEG data. J. of Neurosci. Methods, 164, 177-190.

Morcom, A. M. \& Rugg, M. D. (2012). Retrieval orientation and the control of recollection: an fMRI study. J. Cogn. Neurosci., 24(12), 2372-2384.

Morcom, A. M. (2016). Mind over memory: cuing the aging brain. Curr. Dir. Psych. Sci., 25(3), 143-150.

Morcom, A. M., \& Henson, R. N. A. (2018). Increased Prefrontal Activity with Aging Reflects Nonspecific Neural Responses Rather than Compensation. The Journal of Neuroscience, 38(33), 7303-7313.

Morton, Neal W., and Sean M. Polyn. 2017. "Beta-Band Activity Represents the Recent Past during Episodic Encoding." Neurolmage 147 (February): 692-702. 
Morris, C. D., Bransford, J. D., \& Franks, J. J. (1977). Levels of processing versus transfer appropriate processing. J. Verb. Learn. Verb. Behav., 16, 519533.

Nenert, R., Viswanathan, S., Dubuc, D. M., \& Visscher, K. M. (2012).

Modulations of ongoing alpha oscillations predict successful short-term visual memory encoding. Frontiers in Human Neuroscience, 6, 127.

Norman, K. A., Polyn, S. M., Detre, G. J., \& Haxby, J. V. (2006). Beyond mindreading: multi-voxel pattern analysis of fMRI data. Trends in Cogn. Sci., 10, 424430.

Nyhus, E., \& Curran, T. (2010). Functional role of gamma and theta oscillations in episodic memory. Neuroscience and Biobehavioral Reviews, 34(7), 10231035.

Otten, L. J., Quayle, A. H., Akram, S., Ditewig, T. A., \& Rugg, M. D. (2006). Brain activity before an event predicts later recollection. Nature Neurosci., 9(4), 489491.

Ranganath, C. \& Paller, K. A. (1999). Frontal brain potentials during recognition are modulated by requirements to retrieve perceptual details. Neuron, 22, 605613.

Rebok, G. W., Ball, K., Guey, L. T., Jones, R. N., Kim, H. Y., King, J. W., Marsiske, M., Morris, J. N., Tennstedt, S. L., Unverzagt, F. W., \& Willis, S. L. (2014). Ten-year effects of the advanced cognitive training for independent and 
vital elderly cognitive training trial on cognition and everyday functioning in older adults. J. Amer. Geria. Soc., 62(1), 16-24.

Rissman, J., \& Wagner, A. D. (2012). Distributed representations in memory: insights from functional brain imaging. Annual Review of Psychology, 63, 101128.

Robb, W. G. K. \& Rugg, M. D. (2002). Electrophysiological dissociation of retrieval orientation and retrieval effort. Psychon. Bull. Rev., 9, 583-589.

Rosburg, T. \& Mecklinger, A. (2017). The costs of target prioritization and the external requirements for using a recall-to-reject strategy in memory exclusion tasks: a meta-analysis. Psychon. Bull. Rev., 2017.

Rosburg, T., Johansson, M., Sprondel, V., Mecklinger, A. (2014). Retrieving selfvocalized information: an event-related potential (erp) study on the effect of retrieval orientation. Brain. Cogn. 92c, 123-132.

Rugg, M. D. \& Wilding, E. L. (2000). Retrieval processing and episodic memory. Trends in Cogn. Sci., 4, 108-115.

Rugg, M. D., Allan, K., \& Birch, C. S. (2000). Electrophysiological evidence for the modulation of retrieval orientation by depth of study processing. J. of Cogn. Neurosci., 12, 664-678.

Rugg, M. D. \& Curran, T. (2007). Event-related potentials and recognition memory. Trends. in Cogn. Sci., 11, 251-257. 
Rugg, M. D., Johnson, J. D., Park, H., \& Uncapher, M. R. (2008). Encodingretrieval overlap in human episodic memory: a functional neuroimaging perspective. Progress in Brain Res., 169, 339-352.

Salari, N., \& Rose, M. (2016). Dissociation of the functional relevance of different pre-stimulus oscillatory activity for memory formation. Neurolmage, $125,1013-$ 1021.

Sederberg, P. B., Schulze-Bonhage, A., Madsen, J. R., Bromfield, E. B., McCarthy, D. C., Brandt, A., Tully, M. S., \& Kahana, M. J. (2007). Cerebral Cortex, 17(1), 1190-1196.

Strozak, P., Abedzadeh, D., \& Curran, T. (2016). Separating the FN400 and N400 potentials across recognition memory experiments. Brain Research, 1635, 41-60.

Tulving, E. \& Thompson, D. M. (1973). Encoding specificity and retrieval processes in episodic memory. Psych. Rev., 80, 352-373.

Tulving, E. (1983). Elements of episodic memory. Oxford: Clarendon Press.

Uncapher, M. R., \& Rugg, M. D. (2005). Effects of divided attention on fMRI correlates of memory encoding. J. Cogn. Neurosci., 17(12), 1923-1935.

Villena-González, M., López, V., and Rodríguez, E.(2016). “Orienting Attention to Visual or Verbal/auditory Imagery Differentially Impairs the Processing of Visual Stimuli." Neurolmage 132 (May): 71-78. 
Wilding, E. L., \& Rugg, M. D. (1996). An event-related potential study of recognition memory with and without retrieval of source. Brain, 119, 889-905.

Wilding, E. L. (1999). Separating retrieval strategies from retrieval success: an event-related potential study of source memory. Neuropsychol. 37, 441-454.

Werkle-Bergner, M., Mecklinger, A., Kray, J., Meyer, P., \& Düzel, E. (2005). The control of memory retrieval: Insights from event-related potentials. Cogn. Brain. Res., 24, 599-614. 


\section{VITA}

Mason Price was born in Troy, Missouri in 1988. After graduating from Troy Buchanan High School, he continued his education at the University of Missouri in Columbia. There, he studied Political Science and Psychology, earning a Bachelor of Arts in both disciplines with the anticipation of continuing to an advanced degree in law. However, while taking advantage of an opportunity in psychological research at the University, and under the guidance of a faculty member, Dr. Denis McCarthy, he reversed course and began to pursue a career in academic research. After completing undergraduate studies, he was employed in the Alcohol Cognitions Lab, whic conducted research on the effects of alcohol on cognition. In those two years of employment, he discovered his true passion was the pursuit of knowledge relating to the study of memory using imaging techniques. To this end, he was fortunate enough to be selected by Dr. Jeff Johnson as a PhD candidate in the Memory and Neuroimaging Lab at the University of Missouri. There, he gained invaluable training in statistics, cognitive psychological theory, and imaging technologies. Such training allowed for the continued pursuit of answers regarding fundamental theories of episodic memory and the neural basis of cognition. 